\title{
Multi-wavelength observations of the star forming region in L1616 ${ }^{\star}$
}

\author{
J. M. Alcalá ${ }^{1, \star \star}$, S. Wachter ${ }^{2, \star \star \star}$, E. Covino ${ }^{1}$, M. F. Sterzik ${ }^{3}$, R. H. Durisen ${ }^{4}$, M. J. Freyberg ${ }^{5}$, \\ D. W. Hoard ${ }^{2, \star \star \star}$, and K. Cooksey ${ }^{6, \dagger}$ \\ 1 INAF-Osservatorio Astronomico di Capodimonte, via Moiariello 16, 80131 Napoli, Italy \\ 2 SIRTF Science Center, IPAC/Caltech, M/S220-6, Pasadena, CA 91125, USA \\ 3 European Southern Observatory, Casilla 19001, Santiago 19, Chile \\ ${ }^{4}$ Department of Astronomy, Indiana University, Swain Hall West 319, 727 East 3rd Street, Bloomington, 47405, USA \\ 5 Max-Planck-Institut für extraterrestrische Physik, Giessenbachstrasse, 85748 Garching, Germany \\ ${ }^{6}$ Department of Astronomy and Astrophysics, University of California, Santa Cruz, CA 95064, USA
}

Received 10 October 2003 / Accepted 28 November 2003

\begin{abstract}
We present the results of a multi-wavelength study of the star forming region in L1616. Our observations include ROSAT All-Sky Survey (RASS) and High Resolution Imager (HRI) X-ray observations, optical wide-field imaging and near-IR imaging data and optical long-slit and multi-object spectroscopic follow-up. 22 new low-mass pre-main sequence (PMS) stars are found to be distributed mainly to the East of the L1616 cometary cloud, in about a one-square-degree field. We find that the class-III infrared sources outnumber the class-II infrared sources by a factor of about three. The X-ray properties of the PMS stars in L1616 are quite similar to those of PMS stars detected in the Orion Nebula Cluster. The comparison of the position of the L1616 PMS stars in the HR diagram with theoretical PMS evolutionary tracks yields an average age of 1-2 Myr, with a very small age spread of about 1 Myr. Unlike the fossil star forming regions in Orion, L1616 appears to be a region of on-going star formation relatively far from the Orion A and B clouds. Given the small age spread, the spatial distribution of the PMS stars relative to the head of the cloud, as well as its cometary shape and high star formation efficiency, we conclude that the star formation in L1616 was most likely induced by a single event, the impact of the winds of the massive stars of the Orion OB association or a supernova explosion being the possible triggers. The Initial Mass Function (IMF) in L1616 is roughly consistent with that of the field in the mass range $0.3<M / M_{\odot}<2.5$. Several faint objects, detected in our optical images, are good candidates for young Brown Dwarfs (BDs). We might expect the number of BDs in L1616 to be intermediate between Taurus and the Trapezium.
\end{abstract}

Key words. stars: formation - stars: pre-main sequence - stars: low-mass, brown dwarfs - X-rays: stars ISM: individual objects: L1616

\section{Introduction}

Prior to X-ray and infrared all-sky surveys, almost all of our knowledge regarding low-mass star formation was based on observations of $\mathrm{T}$ associations. Now we know that most lowmass stars form in OB associations (Walter et al. 2000).

\footnotetext{
Send offprint requests to: J.M. Alcalá, e-mail: jmae@na. astro.it

* Based on observations carried out at the European Southern Observatory, La Silla, Chile under proposals numbers 56.E-0566 and 64.I-0355, and at the Calar Alto observatory.

$\star \star$ Visiting Astronomer, Kitt Peak National Observatory, National Optical Astronomy Observatories, which is operated by the Association of Universities for Research in Astronomy, Inc. (AURA) under cooperative agreement with the NSF.

$\star \star \star$ Visiting Astronomer, Cerro Tololo Inter-American Observatory, National Optical Astronomy Observatories, operated by AURA, Inc., under cooperative agreement with the NSF.

$\dagger 2001$ CTIO REU Program student from Valparaiso University.
}

Low-mass star formation in $\mathrm{T}$ and $\mathrm{OB}$ associations may take place differently. In fact, the very different environments of $\mathrm{OB}$ and $\mathrm{T}$ associations may influence the formation of the low-mass stars and subsequent evolution of their circumstellar disks. In the case of OB associations, star formation may be triggered by compression of clouds due to the radiation and winds of OB stars, or supernova shock waves (Lada et al. 1978; Elmegreen et al. 1995; Boss 2003). A consequence of this process is that star formation is sequential. Several cometary clouds observed in the Orion OB association (Ogura \& Sugitani 1998) testify that the strong radiation field from the massive stars in the Belt or Sword regions have an important impact on remnant molecular clouds.

Also, Dolan \& Mathieu (2002) proposed that the ring-like structure of the $\lambda$ Ori region was driven by a supernova explosion about $1 \mathrm{Myr}$ ago.

On the other hand, surveys for $\mathrm{H} \alpha$ emission and X-ray emitting stars in Orion (Nakano et al. 1995 and references 
therein; Sterzik et al. 1995; Alcalá et al. 2000a) revealed many low-mass pre-main sequence (PMS) stars spread throughout the entire Orion OB association. It is not clear whether these stars were formed in small turbulent cloudlets, as proposed by Feigelson (1996), or whether their formation was triggered by the impact of the OB stars or supernova shock waves on a much larger, now dispersed, cloud complex.

In the course of our previous studies of the large scale distribution of ROSAT PSPC ${ }^{1}$ All-Sky Survey (RASS) X-ray sources in the general direction of the Orion star forming region (SFR), several overdensities of X-ray sources with high probability of being low-mas PMS stars were identified (Sterzik et al. 1998; Walter et al. 2000). One density enhancement was found near the reflection nebula NGC 1788, in the L1616 cloud (Lynds 1962). The relatively small width of the enhancement would indicate a diffusion age of less than $5 \mathrm{Myr}$, if these X-ray sources are stars that formed in a central cluster with a dispersion velocity of $2 \mathrm{~km} \mathrm{~s}^{-1}$.

The conspicuous X-ray overdensity detected by the RASS in NGC 1788 (Sterzik et al. 1995) suggested the existence of a small star forming region in the L1616 cloud. Prior to the RASS, the only known young star in the region was the T Tauri star LkH $\alpha$ 333. The Kiso H $\alpha$ survey (Nakano et al. 1995, and references therein) and the optical identifications of the RASS sources (Alcalá et al. 2000a) in Orion provided a few more low-mass PMS stars. Recently, Stanke et al. (2002) reported on millimeter and mid-infrared (MIR) observations of the L1616 region, which revealed five sources, very likely to be associated with very young stellar objects. One of these was an embedded millimeter source, presumably a class 0 protostar, associated with a protostellar jet.

Given the conspicuous X-ray overdensity detected with the RASS in the L1616 cloud, and the possibility that star formation was triggered by stars in the Orion OB association located to the East, we decided to perform multi-wavelength observations in the region, in order to search for other low-mass PMS stars and investigate if there is evidence for sequential star formation. The position of $\operatorname{L1616}\left(\operatorname{RA}(2000) \approx 05^{\mathrm{h}} 05^{\mathrm{m}}\right.$; $\left.\operatorname{Dec}(2000) \approx-03^{\circ}\right)$ relative to the Orion main clouds and its cometary shape can be appreciated in the $100 \mu \mathrm{m}$ IRAS maps (e.g. Fig. 10 by Frasca et al. 2003).

In this paper we report X-ray observations, optical spectroscopy, as well as optical wide-field imaging and nearinfrared observations of the star forming region in L1616.

The paper is outlined as follows: in Sect. 2, we describe the observations and data reduction, while the results of the observations are reported in Sect. 3. Our discussion is presented in Sect. 4 and, finally, our summary and conclusions are presented in Sect. 5.

\section{Observations and data reduction}

\subsection{ROSAT X-ray observations}

The first X-ray observations of L1616 were performed during the RASS in Orion. The L1616 region was detected in

\footnotetext{
${ }^{1}$ PSPC stands for Position-Sensitive Proportional Counter.
}

the analysis by Sterzik et al. (1995) as a significant overdensity of about 4 X-ray sources degree ${ }^{-2}$. Five of the RASS sources (RX J0509.0-0315，RX J0510.1-0427，RX J0510.30330, RX J0511.7-0348, and RX J0512.3-0255) were identified spectroscopically as PMS stars in previous studies in Orion (Alcalá et al. 2000a).

Three additional ROSAT High Resolution Imager (HRI) pointed observations of 18,10 , and $18 \mathrm{ks}$, were performed on 17-20 March 1996, 28 February-6 March 1998, and 25 February-2 March 1998, respectively.

The data were processed using the standard techniques of the Extended Scientific Analysis System (EXSAS) pipeline. The HRI pointings were merged and a source detection algorithm was applied. The sources are identified by an excess of photons in comparison with both a local and a global background using a "maximum likelihood" method (Cruddace et al. 1988). The likelihood is defined as $L=-\ln (P)$, where $P$ is the probability that the observed distribution of photons comes from a spurious background fluctuation. This distribution is estimated using Poisson statistics. The choice of the threshold for a significant source detection depends on how many spurious sources one wants to accept. A conservative value for the detection threshold is 10 which corresponds to $P=4.54 \times 10^{-5}$.

The result of the EXSAS reduction was a list of 27 X-ray sources, but up to five might be spurious. In Table 1 we provide the complete list, but mark those which are likely to be spurious with "no optical counterpart". The radius of the X-ray error circles is typically less than 15 arcsec. In addition to the HRI $\mathrm{X}$-ray sources, we include, at the end of Table 1, four X-ray sources from the RASS Bright Source Catalog (BSC), located outside the field of view of the HRI observations, but still near the L1616 region. The previously known RASS sources identified with PMS stars (Alcalá et al. 2000a) fall outside the area of the HRI pointings.

A search for optical counterparts in the SIMBAD database and in previous lists of PMS stars yields the following results: five X-ray sources are relatively bright HD stars most likely unrelated to the SFR; another five X-ray sources can be identified with young stellar objects previously known to exhibit $\mathrm{H} \alpha$ emission, as well as MIR emission (see Table 1). One of the $\mathrm{H} \alpha$ emission line stars is the T Tauri star $\mathrm{LkH} \alpha-333$, while the other two are Kiso A-0974-16 and Kiso A-0974-18. The MIR sources are from the list reported by Stanke et al. (2002). Finally, the B9V-type star HD 193825, which is believed to produce the reflection nebula in NGC 1788, was not detected in X-rays.

\subsection{Spectroscopic observations}

\subsubsection{Low-resolution spectroscopy}

In order to further identify the remaining X-ray sources, lowresolution long-slit spectra were acquired using the CTIO $1.5 \mathrm{~m}$ telescope on 2001 January 30. In the case of the sources RX J0506.9-0319 and RX J0506.9-0320, two possible optical counterparts inside the X-ray error box were observed. Grating \#16 in the first order was used, resulting in a wavelength coverage of $4750-6750 \AA$ with a resolution of $4.8 \AA$ (FWHM). 
Table 1. ROSAT HRI and PSPC RASS BSC X-ray sources. Column 1: RX J or 1RXS J designations; Cols. 2 and 3: X-ray source coordinates; Col. 4: broad-band count rates; Col. 5: maximum likelihood.

\begin{tabular}{|c|c|c|c|c|c|}
\hline $\mathrm{RXJ} / 1 \mathrm{RXS} \mathrm{J}$ & $\mathrm{RA}(2000)$ & $\operatorname{Dec}(2000)$ & $\operatorname{cts~s}^{-1}$ & $M L$ & Ident \\
\hline $0506.4-0303$ & $05 \quad 06 \quad 26.22$ & $\begin{array}{lll}-03 & 03 & 24.3\end{array}$ & $0.0051 \pm 0.0005$ & 9.3 & no optical counterpart \\
\hline $0507.6-0304$ & $\begin{array}{lll}05 & 07 & 37.49\end{array}$ & $\begin{array}{lll}-03 & 04 & 33.1\end{array}$ & $0.0019 \pm 0.0003$ & 8.3 & no optical counterpart \\
\hline $0506.8-0305$ & $\begin{array}{llll}05 & 06 & 48.71\end{array}$ & $\begin{array}{lll}-03 & 05 & 39.1\end{array}$ & $0.0029 \pm 0.0006$ & 20.2 & \\
\hline $0505.7-0312$ & $\begin{array}{lll}05 & 05 & 41.93\end{array}$ & $\begin{array}{lll}-03 & 12 & 54.1\end{array}$ & $0.0009 \pm 0.0002$ & 8.1 & no optical counterpart \\
\hline $0506.8-0315$ & $\begin{array}{lll}05 & 06 & 44.95\end{array}$ & $\begin{array}{lll}-03 & 15 & 14.6\end{array}$ & $0.0012 \pm 0.0003$ & 23.7 & HD 293814 \\
\hline $0507.4-0317$ & $\begin{array}{lll}05 & 07 & 25.81\end{array}$ & $\begin{array}{lll}-03 & 17 & 11.3\end{array}$ & $0.0016 \pm 0.0004$ & 12.7 & \\
\hline $0506.8-0318$ & $05 \quad 06 \quad 46.60$ & $\begin{array}{lll}-03 & 18 & 01.6\end{array}$ & $0.0014 \pm 0.0003$ & 32.7 & \\
\hline $0507.6-0318$ & $\begin{array}{lll}05 & 07 & 37.48\end{array}$ & $\begin{array}{lll}-03 & 18 & 13.1\end{array}$ & $0.0019 \pm 0.0005$ & 15.3 & \\
\hline $0507.0-0318$ & $05 \quad 06 \quad 56.85$ & $\begin{array}{lll}-03 & 18 & 30.8\end{array}$ & $0.0017 \pm 0.0003$ & 38.1 & \\
\hline $0507.5-0318$ & $\begin{array}{lll}05 & 07 & 29.31\end{array}$ & $\begin{array}{lll}-03 & 18 & 36.4\end{array}$ & $0.0025 \pm 0.0005$ & 40.3 & HD 33056 \\
\hline 0506.9-0319 & $\begin{array}{lll}05 & 06 & 50.72\end{array}$ & $\begin{array}{lll}-03 & 19 & 33.7\end{array}$ & $0.0029 \pm 0.0004$ & 55.9 & L1661 MIR5 \\
\hline $0506.9-0320$ & $05 \quad 06 \quad 54.54$ & $\begin{array}{lll}-03 & 20 & 01.8\end{array}$ & $0.0011 \pm 0.0003$ & 27.1 & $\mathrm{LkH} \alpha 333=$ Kiso A-0974-14 \\
\hline $0507.4-0320$ & $\begin{array}{lll}05 & 07 & 22.13\end{array}$ & $\begin{array}{lll}-03 & 20 & 14.1\end{array}$ & $0.0011 \pm 0.0003$ & 17.1 & \\
\hline $0506.5-0320$ & $\begin{array}{lll}05 & 06 & 27.61\end{array}$ & $\begin{array}{lll}-03 & 20 & 23.9\end{array}$ & $0.0173 \pm 0.0010$ & 964.9 & \\
\hline $0506.9-0320$ & $05 \quad 0652.97$ & $\begin{array}{lll}-03 & 20 & 50.0\end{array}$ & $0.0023 \pm 0.0004$ & 56.5 & L1661 MIR1, L1661 MIR2 \\
\hline $0506.1-0321$ & $\begin{array}{lll}05 & 06 & 03.29\end{array}$ & $\begin{array}{lll}-03 & 21 & 05.1\end{array}$ & $0.0016 \pm 0.0004$ & 13.1 & HD 293771 \\
\hline $0507.1-0321$ & $\begin{array}{lll}05 & 07 & 06.08\end{array}$ & $\begin{array}{lll}-03 & 21 & 26.3\end{array}$ & $0.0013 \pm 0.0003$ & 32.6 & Kiso A-0974-16 \\
\hline $0505.6-0322$ & $\begin{array}{lll}05 & 05 & 37.86\end{array}$ & $-03 \quad 22 \quad 45.6$ & $0.0039 \pm 0.0004$ & 99.3 & HD 293772 \\
\hline $0506.5-0323$ & $\begin{array}{lll}05 & 06 & 27.48\end{array}$ & $\begin{array}{lll}-03 & 23 & 00.0\end{array}$ & $0.0035 \pm 0.0005$ & 100.7 & HD 32911 \\
\hline $0506.4-0323$ & $\begin{array}{lll}05 & 06 & 25.98\end{array}$ & $\begin{array}{lll}-03 & 23 & 24.4\end{array}$ & $0.0088 \pm 0.0006$ & 284.3 & \\
\hline $0507.2-0323$ & $\begin{array}{lll}05 & 07 & 10.85\end{array}$ & $\begin{array}{lll}-03 & 23 & 50.9\end{array}$ & $0.0028 \pm 0.0004$ & 67.8 & Kiso A-0974-18 \\
\hline $0507.3-0326$ & $\begin{array}{lll}05 & 07 & 14.87\end{array}$ & $\begin{array}{lll}-03 & 26 & 44.4\end{array}$ & $0.0044 \pm 0.0006$ & 93.6 & \\
\hline $0506.8-0327$ & $05 \quad 06 \quad 48.15$ & $\begin{array}{lll}-03 & 27 & 37.0\end{array}$ & $0.0024 \pm 0.0004$ & 47.8 & \\
\hline $0506.4-0328$ & 050623.63 & $\begin{array}{lll}-03 & 28 & 48.9\end{array}$ & $0.0015 \pm 0.0003$ & 9.4 & \\
\hline $0506.2-0330$ & $\begin{array}{lll}05 & 06 & 12.93\end{array}$ & $\begin{array}{lll}-03 & 30 & 49.8\end{array}$ & $0.0019 \pm 0.0003$ & 8.0 & no optical counterpart \\
\hline $0506.3-0335$ & $\begin{array}{lll}05 & 06 & 15.42\end{array}$ & $\begin{array}{lll}-03 & 35 & 13.9\end{array}$ & $0.0030 \pm 0.0004$ & 9.8 & no optical counterpart \\
\hline $0506.6-0337$ & $\begin{array}{lll}05 & 06 & 34.27\end{array}$ & $\begin{array}{lll}-03 & 37 & 09.0\end{array}$ & $0.0057 \pm 0.0008$ & 41.0 & \\
\hline $045912.4-033711$ & $04 \quad 59 \quad 12.40$ & $\begin{array}{lll}-03 & 37 & 11.5\end{array}$ & $0.0345 \pm 0.0119$ & 9.0 & PSPC RASS BSC source \\
\hline 050416.9-021426 & $\begin{array}{lll}05 & 04 & 16.90\end{array}$ & $\begin{array}{lll}-02 & 14 & 26.0\end{array}$ & $0.0339 \pm 0.0108$ & 18.0 & PSPC RASS BSC source \\
\hline $051011.5-025355$ & $\begin{array}{lll}05 & 10 & 11.50\end{array}$ & $-02 \quad 53 \quad 55.0$ & $0.0390 \pm 0.0114$ & 21.0 & PSPC RASS BSC source \\
\hline $051043.2-031627$ & $\begin{array}{lll}05 & 10 & 43.20\end{array}$ & $\begin{array}{lll}-03 & 16 & 27.5\end{array}$ & $0.0376 \pm 0.0126$ & 14.0 & PSPC RASS BSC source \\
\hline
\end{tabular}

Exposure times ranged from 120 to 900 s. Arc lamp spectra for wavelength calibration were obtained before and after each exposure. Bias and flat-field corrections to the raw spectrum images were performed in the standard manner using $\mathrm{IRAF}^{2}$. The IRAF package CTIOSLIT was used to extract and wavelength/flux calibrate the spectra. The wavelength-scale zeropoint was confirmed through comparison with the positions of the night-sky lines. The instrumental response function was successfully removed from the target spectra using various standard stars, however, no attempt was made to correct the absolute flux levels for slit losses.

${ }^{2}$ IRAF is distributed by the National Optical Astronomy Observatory, which is operated by the Association of the Universities for Research in Astronomy, Inc., under contract with the National Science Foundation.
The four sources from the RASS BSC were investigated in November 1995, using the Boller \& Chivens spectrograph at the ESO $1.5 \mathrm{~m}$ telescope. The covered spectral range was $4700-6950 \AA$ at a resolution of about $3.5 \AA$ (FWHM). The data reduction was performed as described in Alcalá et al. (1995). In Fig. 1 some examples of low-resolution spectra are shown.

\subsubsection{Intermediate-resolution spectroscopy}

Intermediate-resolution spectroscopy was also performed in January 2000 using the multi-fibre spectrograph HYDRA, attached to the WIYN ${ }^{3} 3.5 \mathrm{~m}$ telescope at Kitt Peak.

\footnotetext{
3 The WIYN Observatory is a joint facility of the University of Wisconsin-Madison, Indiana University, Yale University, and the National Optical Astronomy Observatory.
} 


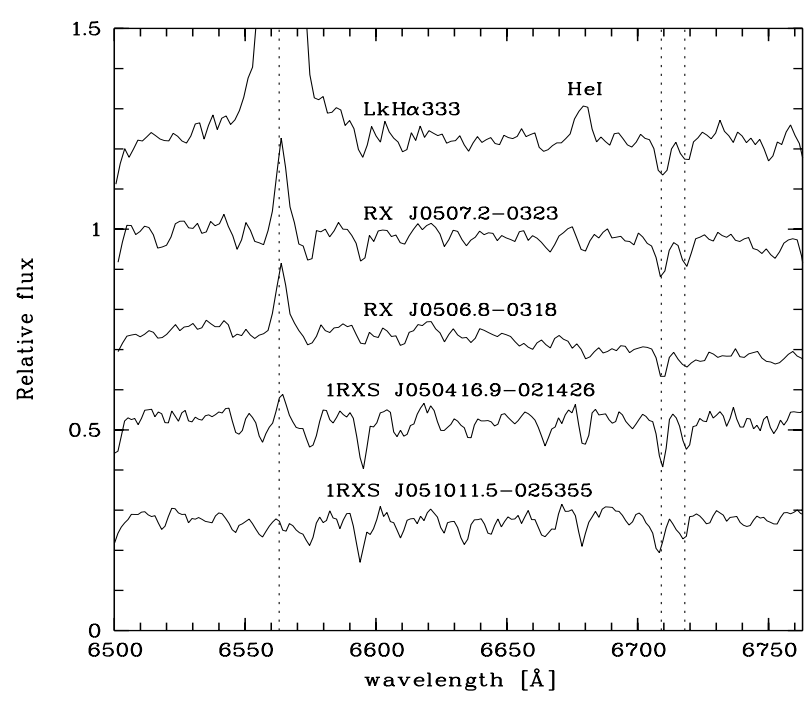

Fig. 1. Examples of low-resolution spectra obtained at CTIO and ESO. The dotted lines represent the position of the $\mathrm{H} \alpha$, Li I $\lambda 6708 \AA$ and Ca I $\lambda 6718 \AA$ lines respectively. The spectrum of $\mathrm{LkH} \alpha 333$ shows the $\mathrm{He} \mathrm{I} \lambda 6678 \AA$ in emission.

The 860@30.6 grating in the second order, centered at $6600 \AA$, was used together with the KG-3+GG-495 filters to separate overlapping orders. The resulting spectra cover a wavelength range of $6100-7000 \AA$. The nominal spectral resolution, measured from several isolated lines of the Th-Ar comparison spectrum, was about $1.5 \AA$ ( $F W H M)$. At such resolution, the $\mathrm{Li}$ $\lambda 6708 \AA$ line is well resolved from the Ca $\lambda 6718 \AA$ absorption line and measurements of the $\mathrm{Li}$ equivalent width can be performed with an average error of less than $50 \mathrm{~m} \AA$, depending on the $\mathrm{S} / \mathrm{N}$ ratio of the spectra and the spectral type (see Covino et al. 1997).

The observational strategy was to perform an unbiased lithium survey. We used the USNO catalog as reference for the fibre positions. 1072 objects were extracted from the USNO catalog in the magnitude range $11<R_{\mathrm{USNO}}<16$ and in a circular area with a diameter of $1^{\circ}$ in L1616. Because of bad weather, only five out of the seven HYDRA configurations in L1616 could be performed. Therefore, the survey is not complete. The exposure times ranged from $900 \mathrm{~s}$ to $1800 \mathrm{~s}$, depending on the magnitude of the objects.

A grid of stars with different spectral types in the Praesepe cluster were observed with the same instrumental set-up, in order to determine radial velocities by means of the crosscorrelation technique (see Sect. 3.3).

The data reduction was accomplished with the dohydra task in IRAF. The reduction consisted of the overscan and bias subtraction, identification of the different apertures using the internal flat-field image (obtained immediately after each science exposure), flat-fielding, wavelength calibration using the Th-Ar comparison spectrum (obtained immediately after each science exposure), and extraction of the one-dimensional spectra. No standard stars for flux calibration were observed.

Except for the X-ray sources RXJ0506.9-0319 and RX J0506.9-0320, practically all the sources investigated with the CTIO $1.5 \mathrm{~m}$ telescope were also observed with HYDRA. Some examples of HYDRA spectra are shown in Fig. 2.

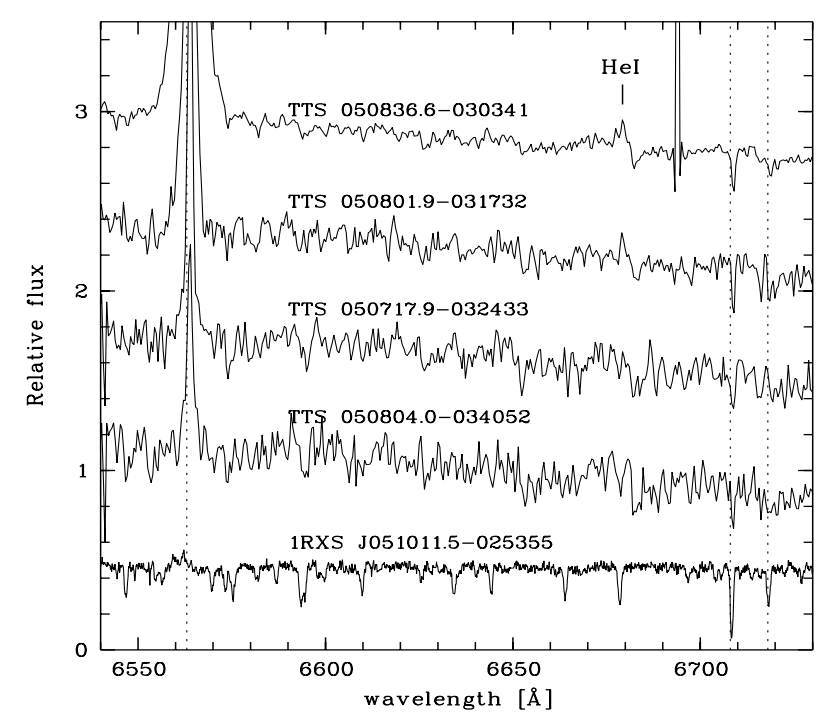

Fig. 2. Examples of HYDRA spectra and a high-resolution FOCES spectrum (bottom). The dotted lines are as in Fig. 1. The spectra of TTS 050836.6-30341 and TTS 050801.9-31732 show the He I $\lambda 6678 \AA$ in emission.

A total of 423 useful spectra (i.e. $40 \%$ of the sample extracted from the USNO catalog) were obtained with HYDRA, but only the stars with lithium absorption are considered here. Since the HYDRA observations were unbiased toward X-ray detection, they can also provide new low-mass PMS stars not detected in the X-ray observations.

\subsubsection{High-resolution spectroscopy}

The four RASS BSC sources were also investigated at high spectral resolution $(\lambda / \Delta \lambda \approx 30000)$ in October 1997 using the Fiber Optics Cassegrain Echelle Spectrograph (FOCES; Pfeiffer et al. 1998) attached to the $2.2 \mathrm{~m}$ telescope at the Calar Alto Observatory in Spain. The technical details of these observations, the data reduction, as well as their analysis are described in Alcalá et al. (2000a). An example of a highresolution spectrum is shown at the bottom of Fig. 2.

\subsubsection{Summary of spectroscopic results}

In summary, 14 of the 22 HRI X-ray sources detected with a high confidence level were investigated spectroscopically at CTIO or with HYDRA. These include also four of the HD stars. One of the remaining 8 HRI X-ray sources detected with high confidence can be identified with HD 293772, which is unrelated to the SFR. Therefore, there remain 7 interesting HRI $\mathrm{X}$-ray sources which still lack spectroscopy, but based on our photometry (see below), six of them are very good candidates for low-mass PMS stars (see Appendix A). Also, based on our wide-field imaging data (see below), we find no optical counterparts to the sources RX J0506.4-0303, RX J0507.6-0304, RX J0505.7-0312, RX J0506.2-0330 and RX J0506.3-0335.

The CTIO and ESO spectra were mainly used for the lithium detection and for the spectral type classification, while 
Table 2. Photometric zero points and color terms.

\begin{tabular}{ccc}
\hline \hline Band & Z.P. & Col. term \\
\hline$B$ & 24.78 & +0.42 \\
$V$ & 24.01 & -0.16 \\
$R_{\mathrm{C}}$ & 24.42 & -0.03 \\
$I_{\mathrm{C}}$ & 23.16 & +0.11 \\
\hline
\end{tabular}

the HYDRA and Calar Alto spectra were used to measure Li $\lambda 6708 \AA$ equivalent widths and radial velocities.

\subsection{Photometric data}

\subsubsection{Optical Wide-Field imaging}

CCD mosaic imaging in the $B, V, R$, and $I$ bands was obtained with the Wide-Field Imager (WFI) at the ESO/MPIA $2.2 \mathrm{~m}$ telescope at ESO La Silla on 26 March 2000 under photometric conditions. In order to cover the gaps between the CCDs of the mosaic, we performed a sequence of five dithered positions in each filter. The exposure time of each dithered image was $120 \mathrm{~s}$, amounting to a total exposure time of $10 \mathrm{~min}$ in each filter. The resulting mosaic covers about $900 \mathrm{arcmin}^{2}$.

The Landolt fields Lan-98, Lan-107 and Lan-110, as well as stars from the extended catalog of Landolt standards provided by Stetson in the CADC (Canadian Astronomy Data Center) Web page ${ }^{4}$ were observed for the broad-band photometric calibration.

The data were reduced using the mscred task under IRAF, following the methods described in Alcalá et al. (2002). The reduction consisted of the bias correction, flat-fielding using a super-flat image, fringing correction (for the $R$ and $I$ filters), astrometric solution, and image stacking. The absolute astrometric precision is imposed by the reference astrometric catalog. In our case we use the USNO A2 catalog (Monet et al. 1998), which allows an absolute rms accuracy of $\sim 0.3^{\prime \prime}$.

The photometric zero points and color terms were determined using the Landolt standards. Average extinction coefficients for the ESO-La Silla observatory were adopted for the airmass corrections. The transformation equations used are:

$B=b+C_{B} \cdot(b-v)+Z_{B}$,

$V=v+C_{V} \cdot(b-v)+Z_{V}$,

$R_{\mathrm{C}}=r+C_{R} \cdot(v-r)+Z_{R}$,

$I_{\mathrm{C}}=i+C_{I} \cdot(v-i)+Z_{I}$,

where $b, v, r, i$ are the instrumental magnitudes corrected for atmospheric extinction, $C_{B}, C_{V}, C_{R}, C_{I}$ are the color terms, and $Z_{B}, Z_{V}, Z_{R}, Z_{I}$ are the zero points in the $B, V, R, I$ bands respectively. The resulting photometric zero points and color terms are reported in Table 2 .

The rms of the fits of Eqs. (1-4) are 0.034, 0.025, 0.030 and 0.025 respectively.

The stellar objects above the $3 \sigma$ level were extracted using both SExtractor (Bertin \& Arnouts 1996) and Daophot

\footnotetext{
${ }^{4}$ http://cadcwww.hia.nrc.ca/standards
}

(Stetson 1987), with consistent results. More than 7000 objects were extracted from the $R$-band image above the $3 \sigma$ level. The airmass corrected aperture instrumental magnitudes were then transformed to the Johnson-Cousins photometric system using the above transformation equations.

The estimated completeness and limiting magnitudes in the $V$ band are 21.0 and 22.0 respectively.

The optical magnitudes for some of the newly identified PMS stars are listed in Table 3.

\subsubsection{MASS data}

In addition to the optical WFI data, we retrieved infrared (IR) data from the Two Micron All-Sky Survey (2MASS) data release. All the stars identified as lowmass PMS stars during the spectroscopic observations (see Sect. 3.1) were found to have a match in the 2MASS point source catalog (which can be accessed electronically at http://irsa.ipac.caltech.edu/). All these objects were detected in the 2MASS with a high confidence level. The mean errors in the 2MASS photometry are $0.03,0.02$ and 0.03 in the $J, H$, and $K_{\mathrm{S}}$ bands respectively. The $J, H, K_{\mathrm{S}}$ photometry of the confirmed PMS stars in L1616 (see Sect. 3.1) is provided in Table 3.

\subsubsection{Near-infrared imaging}

Near-infrared (NIR) imaging in the $J, H$, and $K$ bands of the core of L1616 was performed with the CTIO $1.5 \mathrm{~m}$ telescope and OSIRIS on 5 March 2001. These observations cover a field of view of about $5^{\prime} \times 5^{\prime}$ centered at $\mathrm{RA}(2000)=05^{\mathrm{h}} 06^{\mathrm{m}} 54.3$; and $\operatorname{Dec}(2000)=-03^{\circ} 20^{\prime} 16^{\prime \prime}$ and provide improved spatial resolution $\left(\approx 1^{\prime \prime} .1\right.$ seeing with a scale of $0.46 /$ pixel $)$, as well as deeper magnitudes than those provided by the 2MASS images. This improves the NIR photometry in the case of close pairs like RX J0506.9-0319 and RX J0506.9-0320.

For each filter, a sky frame was first constructed from the 9 dithered images with an exposure time of $3 \mathrm{~s}$ each. After the sky subtraction was applied to each object frame, the data were flatfielded with a normalized dome flat. Finally, the images were shifted and combined into a single frame for each filter. An astrometric solution for the frames was derived from comparison to 2MASS source positions.

\section{Results}

\subsection{New pre-main sequence stars}

Based on the spectroscopic observations, we have newly identified 22 stars with strong Li I $\lambda 6708 \AA$ absorption, spectral type later than about G5 and, in most cases, the $\mathrm{H} \alpha$ line well in emission. Although three of these stars were detected in the previous MIR observations by Stanke et al. (2002) and another three were revealed in the Kiso $\mathrm{H} \alpha$ survey, the first evidence for lithium and late type spectrum is provided in this work. Therefore, we classify all these stars as new $\mathrm{T}$ Tauri stars. Further arguments on the PMS nature of these stars are presented in the discussion section. 
Table 3. Pre-main sequence stars in L1616 and their photometry.

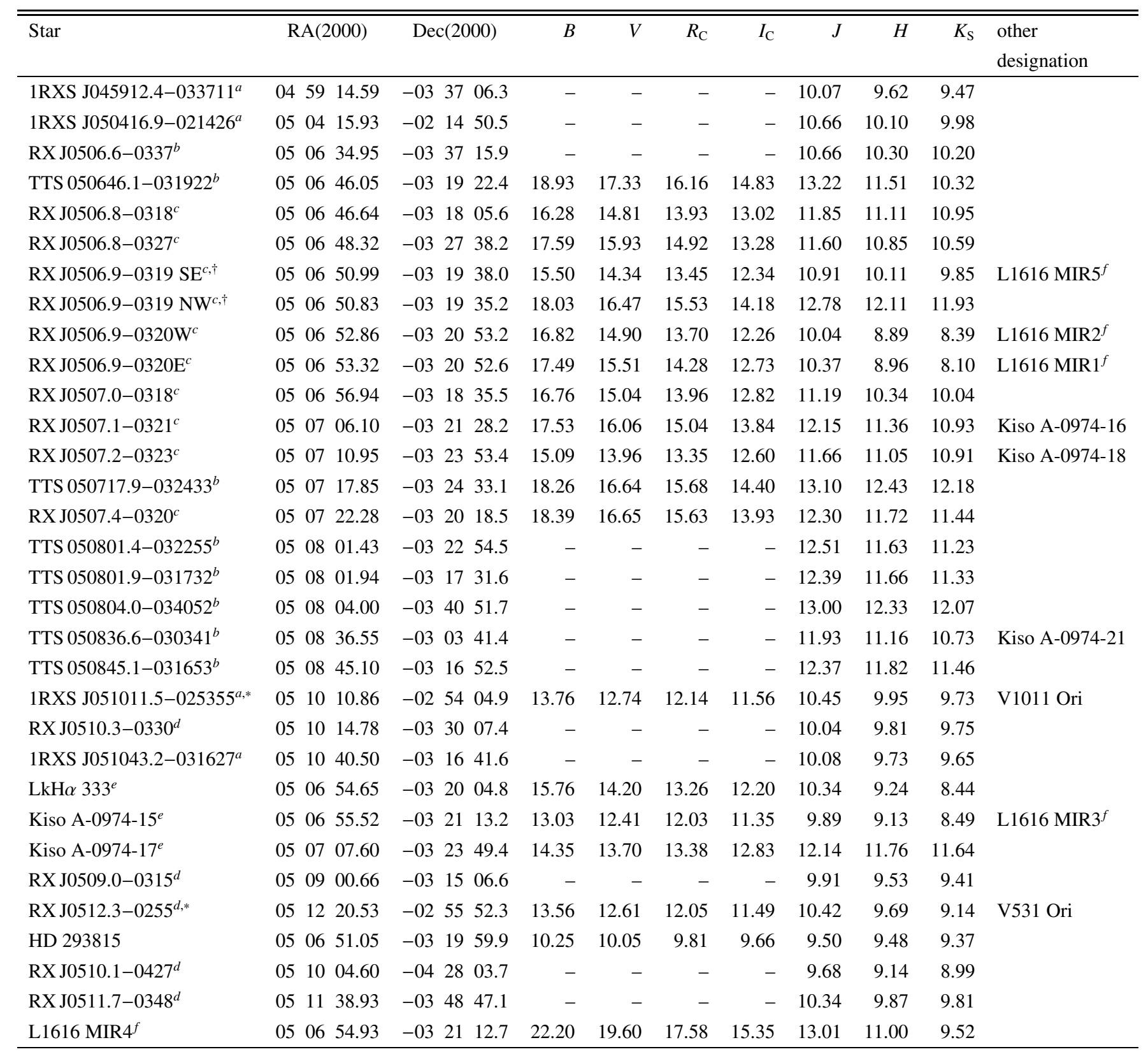

$\dagger$ CTIO NIR photometry (see Sect. 2.3.3); * averaged optical photometry taken from Cieslinski et al. (1997).

${ }^{a}$ RASS BSC + Calar Alto spectra (this work); ${ }^{b}$ HYDRA-WIYN spectra (this work); ${ }^{c}$ ROSAT-HRI + CTIO or HYDRA-WIYN spectra (this work); ${ }^{d}$ previously known RASS WTTS by Alcalá et al. (2000a); ${ }^{e}$ previously known H $\alpha$ emission-line star; ${ }^{f}$ MIR source by Stanke et al. (2002).

If we assume that the B9V-type star HD 293815 is a member of the cluster, and also counting the millimeter source found by Stanke et al. (2002), then the number of young stellar objects known so far in L1616 is 33 . The coordinates and $B, V, R_{\mathrm{C}}, I_{\mathrm{C}}, J, H, K_{\mathrm{S}}$ magnitudes of these objects are listed in Table 3. We have also included in this table the previously known PMS stars and HD 293815. The coordinates provided in Table 3 are those from the 2MASS catalog, which have a precision of about 0.1 arcsec.

In total, 21 PMS stars were detected in X-rays, while 7 additional PMS stars, not detected in X-rays, were found with
HYDRA. Other weak-lithium $(W(\mathrm{Li})<200 \mathrm{~m} \AA)$ stars found with HYDRA are reported in Appendix B. The objects revealed with HYDRA, but not detected in X-rays, are designated with "TTS" followed by their position, according to the IAU convention.

Twelve of the spectroscopically confirmed $\mathrm{T}$ Tauri stars plus three previously known $\mathrm{H} \alpha$ emission-line stars (LkH $\alpha$ 333, Kiso A-0974-15 and Kiso A-0974-17) and the MIR source L1616 MIR4, fall in the WFI images. The $B, V, R_{\mathrm{C}}$ and $I_{\mathrm{C}}$ photometry of these stars is reported in Table 3, together with their NIR photometry from the 2MASS and the CTIO NIR 


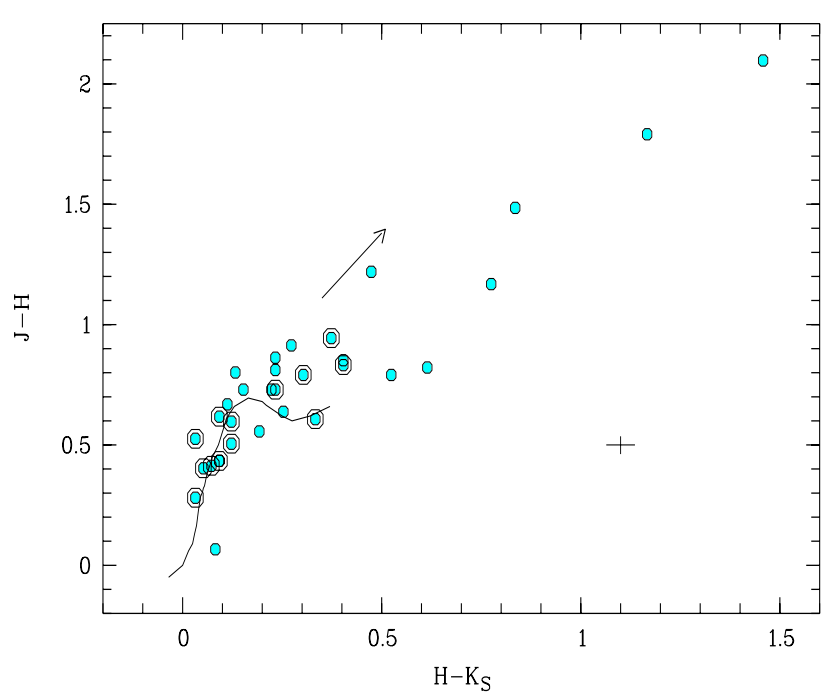

Fig. 3. IR color-color diagram of the PMS stars in L1616. The solid line represents the colors of normal field dwarfs from Bessell \& Brett (1988). The 2MASS magnitudes were homogenized to the Bessell \& Brett system using the transformation equations by Carpenter (2001). The arrow indicates the normal reddening vector. The encircled dots represent the stars with NIR photometry only. The average errors are indicated in the lower right.

observations. The stars lacking optical photometry fall outside the sky area investigated with the WFI, but for RX J0512.30255 and 1RXS J051011.5-025355 we retrieved the optical photometry from Cieslinski et al. (1997). In total, 18 of the PMS stars have optical and NIR photometry. Unfortunately, the star HD 293815 is saturated in our WFI images. Therefore, we use the photometry from the literature (Neckel \& Chini 1980).

The NIR color-color diagram of the PMS stars in L1616 is shown in Fig. 3. The stars TTS 050646.1-031922, RX J0506.90320W, RX J0506.9-0320E, LkH $\alpha 333$ and L1616 MIR4 show very red colors, possibly due to strong infrared excess. The 13 stars with NIR photometry only (represented by the encircled dots in Fig. 3) do have less red colors. Among these stars TTS 050801.4-032255 and TTS 050836.6-030341 still have colors which indicate possible infrared excess. The rest of the stars have more or less normal colors.

In the following sections we use the spectroscopic and photometric data in order to determine the stellar parameters for the PMS stars reported in Table 3. For 18 PMS stars we can determine spectral energy distributions from the optical and NIR photometry, while for the others we use the spectral information and the 2MASS data to derive their stellar parameters.

\subsection{Spectral types and effective temperatures}

Spectral types (SpT) were assigned using the low-resolution spectra obtained at CTIO and a grid of standard spectra with similar resolution as the PMS star spectra. The procedure described in Covino et al. (1997) and Alcalá et al. (2000a) was applied for the spectral type classification. In the cases where no low-resolution spectrum is available, we use the Praesepe stars as templates for the spectral type classification. We estimate that the spectral type classification is precise to within less than two sub-classes.

Unfortunately, the stars Kiso A-0974-15, Kiso A-0974-17 and L1616 MIR4 lack spectroscopic observations and hence, we cannot determine their spectral type directly (but see Sect. 3.6 below).

An estimate of the effective temperature can be obtained using the calibration between spectral type and effective temperature by de Jager \& Nieuwenhuijzen (1987) for luminosity class $V$. The derived effective temperatures are consistent, within the errors, with those derived using the calibrations between the $\mathrm{Na}$ I D lines equivalent width and $\log T_{\text {eff }}$ for luminosity class $\mathrm{V}$ given by Tripicchio et al. (1997).

For the star HD 293815, we use the spectral type B9V provided in the literature (Neckel \& Chini 1980) in order to derive the effective temperature using the same temperature scale.

The spectral types are reported in Table 4, while the derived effective temperatures are reported in Table 5.

\subsection{Radial velocity determinations}

We used the intermediate-resolution HYDRA spectra and the high-resolution Calar Alto spectra in order to measure heliocentric radial velocities $(R V)$. Despite the intermediate resolution of the HYDRA spectra of about $1.5 \AA F W H M$, reasonable results are obtained by means of cross-correlation analysis. The cross-correlation of the target and template spectra was performed after rebinning the spectra to a logarithmic wavelength scale, in order to eliminate the dependence of the Doppler shift on wavelength. For this purpose we used the fxcor task under IRAF. The templates used were stars in the Praesepe cluster, obtained with the same HYDRA instrumental set-up as the targets. For the Calar Alto spectra we also used the cross-correlation analysis, as described in Alcalá et al. (2000a). In both cases, only parts of the spectra free of emission lines and/or not affected by telluric absorption lines were considered. Therefore, the $\mathrm{H} \alpha$ line was excluded from the cross-correlation analysis. We estimate average errors of $5 \mathrm{~km} \mathrm{~s}^{-1}$ and $1.5 \mathrm{~km} \mathrm{~s}^{-1}$ in the $R V$ measurements from the HYDRA and Calar Alto spectra, respectively.

Both the M 3 type stars TTS 050646.1-031922 and TTS 050845.1-031653, were observed with HYDRA, but the spectra have a low S/N. In the case of the former, the crosscorrelation still provides reliable results within the average error, while for the latter the radial velocity is not very reliable. The cross-correlation peak for the star RX J0506.8-0318 shows some indication for binarity. However, this must be confirmed with higher resolution spectroscopy. The heliocentric radial velocities are reported in Table 4 . No radial velocity measurements were attempted from the low-resolution spectra.

\subsection{H $\alpha$ and Li equivalent widths and Li abundances}

$\mathrm{H} \alpha$ and $\mathrm{Li}$ I $\lambda 6708 \AA$ line equivalent widths, $W(\mathrm{H} \alpha)$ and $W(\mathrm{Li})$, respectively, were determined by integration of the lines, interpolating linearly the continuum on both sides of the corresponding line. We adopted the convention that positive 
Table 4. Spectral types, $\mathrm{H} \alpha$ and lithium equivalent widths, $W(\mathrm{H} \alpha), W(\mathrm{Li})$, lithium abundances, $N(\mathrm{Li})$, and radial velocities, $R V$, for the PMS stars in L1616.

\begin{tabular}{|c|c|c|c|c|c|c|c|}
\hline Star & $\mathrm{SpT}$ & $\begin{array}{c}W(\mathrm{H} \alpha) \\
{[\AA]}\end{array}$ & $\begin{array}{l}W(\mathrm{Li}) \\
{[\mathrm{m} \AA]}\end{array}$ & $N(\mathrm{Li})$ & $\begin{array}{c}R V \\
{\left[\mathrm{~km} \mathrm{~s}^{-1}\right]}\end{array}$ & Type & notes \\
\hline 1RXS J045912.4-033711 & G7 & $1.80 \pm 0.20$ & $355 \pm 10$ & 3.50 & $+12.2 \pm 1.6$ & $\mathrm{~W}$ & \\
\hline 1RXS J050416.9-021426 & $\mathrm{K} 3$ & $0.03 \pm 0.20$ & $475 \pm 10$ & 3.25 & $+19.8 \pm 1.5$ & $\mathrm{~W}$ & \\
\hline RX J0506.6-0337 & $\mathrm{K} 3$ & $1.00 \pm 0.20$ & $299 \pm 10$ & 2.59 & $+19.0 \pm 4.0$ & $\mathrm{~W}$ & \\
\hline TTS 050646.1-031922 & M3 & $-60.85 \pm 5.00$ & - & - & $+26.0 \pm 5.0$ & $\mathrm{C}$ & noisy spectrum \\
\hline RX J0506.8-0318 & M0 & $-1.30 \pm 0.20$ & $770 \pm 30$ & 2.65 & $+23.0 \pm 5.0$ & $\mathrm{~W}$ & binary ? $W_{\text {CTIO }}(\mathrm{H} \alpha)=-1.3$ \\
\hline RX J0506.8-0327 & M4 & $-8.50 \pm 0.20$ & $650 \pm 25$ & 2.21 & $+27.0 \pm 2.0$ & $\mathrm{~W}$ & \\
\hline RX J0506.9-0319 $\mathrm{SE}^{A}$ & K6 & $-2.60 \pm 0.20$ & $450 \pm 100$ & 2.62 & - & $\mathrm{W}$ & \\
\hline RX J0506.9-0319 NW ${ }^{A}$ & M2 & $-4.90 \pm 0.30$ & $400 \pm 150$ & 1.44 & - & $\mathrm{W}$ & \\
\hline RX J0506.9-0320W ${ }^{A}$ & $\mathrm{~K} 3$ & $-1.80 \pm 0.20$ & $470 \pm 100$ & 3.23 & - & $\mathrm{W}$ & \\
\hline RXJ0506.9-0320E ${ }^{A}$ & $\mathrm{~K} 5$ & $-1.60 \pm 0.20$ & $230 \pm 100$ & 1.98 & - & $\mathrm{W}$ & \\
\hline RX J0507.0-0318 & M1 & $-1.80 \pm 0.20$ & $650 \pm 25$ & 2.29 & $+29.0 \pm 2.0$ & $\mathrm{~W}$ & $W_{\text {Стіо }}(\mathrm{H} \alpha)=-1.6$ \\
\hline RX J0507.1-0321 & M1 & $-15.50 \pm 0.50$ & $620 \pm 25$ & 2.24 & $+22.0 \pm 2.0$ & $\mathrm{C}$ & $W_{\mathrm{CTIO}}(\mathrm{H} \alpha)=-72.9$ \\
\hline RXJ0507.2-0323 & $\mathrm{K} 7$ & $-0.90 \pm 0.20$ & $540 \pm 30$ & 2.70 & $+24.0 \pm 2.0$ & W & $W_{\text {СТIO }}(\mathrm{H} \alpha)=-1.2$ \\
\hline TTS 050717.9-032433 & M2 & $-4.50 \pm 0.50$ & $530 \pm 30$ & 1.90 & $+30.0 \pm 5.0$ & $\mathrm{~W}$ & \\
\hline RX J0507.4-0320 & M5 & $-5.50 \pm 2.00$ & $630 \pm 40$ & 2.19 & $+26.0 \pm 6.0$ & $\mathrm{~W}$ & \\
\hline TTS 050801.4-032255 & M3 & $-21.50 \pm 0.50$ & $510 \pm 25$ & 1.71 & $+18.0 \pm 4.0$ & $\mathrm{C}$ & \\
\hline TTS 050801.9-031732 & M3 & $-15.50 \pm 0.50$ & $410 \pm 25$ & 1.30 & $+23.0 \pm 5.0$ & $\mathrm{C}$ & \\
\hline TTS 050804.0-034052 & M4 & $-2.20 \pm 0.20$ & $410 \pm 20$ & 1.12 & $+15.0 \pm 5.0$ & $\mathrm{~W}$ & \\
\hline TTS $050836.6-030341$ & M5 & $-68.50 \pm 1.00$ & $560 \pm 30$ & 1.78 & $+27.0 \pm 6.0$ & $\mathrm{C}$ & \\
\hline TTS $050845.1-031653$ & M3 & $-5.50 \pm 1.00$ & - & - & $+3.0 \pm 5.0$ & $\mathrm{~W}$ & noisy spectrum \\
\hline 1RXS J051011.5-025355 & $\mathrm{K} 1$ & $-0.10 \pm 0.20$ & $415 \pm 10$ & 3.36 & $+26.1 \pm 1.5$ & $\mathrm{~W}$ & \\
\hline RX J0510.3-0330 & G8 & $1.50 \pm 0.20$ & $320 \pm 10$ & 3.27 & $+18.3 \pm 2.1$ & $\mathrm{~W}$ & \\
\hline 1RXS J051043.2-031627 & G5 & $2.40 \pm 0.20$ & $235 \pm 10$ & 3.19 & $+20.1 \pm 2.6$ & $\mathrm{~W}$ & \\
\hline $\mathrm{LkH} \alpha 333$ & K6 & $-62.00 \pm 1.00$ & $420 \pm 30$ & 2.53 & $+27.0 \pm 5.0$ & $\mathrm{C}$ & $W_{\text {СтIо }}(\mathrm{H} \alpha)=-50.8$ \\
\hline Kiso A-0974-15 & K5: & - & - & - & - & $?$ & \\
\hline Kiso A-0974-17 & K3: & - & - & - & - & $?$ & \\
\hline RX J0509.0-0315 & $\mathrm{K} 1$ & $1.10 \pm 0.20$ & $320 \pm 10$ & 2.97 & $+23.0 \pm 2.3$ & $\mathrm{~W}$ & \\
\hline RX J0512.3-0255 & $\mathrm{K} 1$ & $-6.50 \pm 0.50$ & $440 \pm 10$ & 3.48 & $+22.7 \pm 1.5$ & $\mathrm{~W}$ & \\
\hline HD 293815 & B9 & & & & & & \\
\hline RXJ0510.1-0427 ${ }^{B}$ & $\mathrm{~K} 4$ & $-0.20 \pm 0.20$ & $230 \pm 10$ & 2.15 & $+21.4 \pm 1.0$ & $\mathrm{~W}$ & \\
\hline RX J0511.7-0348 ${ }^{B}$ & $\mathrm{~K} 1$ & $1.80 \pm 0.20$ & $370 \pm 15$ & 3.16 & $+16.3 \pm 1.7$ & $\mathrm{~W}$ & \\
\hline L1616 MIR4 & M6: & - & - & - & - & $?$ & \\
\hline
\end{tabular}

${ }^{A}$ Values of $W(\mathrm{H} \alpha)$ and $W(\mathrm{Li})$ determined from low-resolution spectra.

${ }^{B}$ Spectral types, $W(\mathrm{Li}), N(\mathrm{Li})$ and $R V$ taken from Alcalá et al. (2000a).

equivalent widths indicate absorption lines. The $\mathrm{H} \alpha$ and lithium equivalent widths are reported in Table 4.

The main source of error on these measurements comes from the uncertainty in the placement of the photospheric continuum. For each spectrum, at least three individual measurements of $W(\mathrm{H} \alpha)$ and $W(\mathrm{Li})$ were obtained by setting the continuum at different positions. In the case of the HYDRA and FOCES spectra, the mean estimated error on $W(\mathrm{Li})$ is $30 \mathrm{~m} \AA$ in most cases, while for $W(\mathrm{H} \alpha)$ the relative error is about $10 \%$. For the stars later than M1, in which the continuum placement is difficult because of photospheric absorption bands, the uncertainty of $W(\mathrm{Li})$ may be as high as $50 \mathrm{~m} \AA$. We also measured $W(\mathrm{Li})$ from the low-resolution spectra but these values may be overestimated because of blends with nearby iron lines. Hence, except for the stars RXJ0506.9-0319 SE, RX J0506.9-0319 NW, RX J0506.9-0320W and RX J0506.9-0320E, which were observed only at low-resolution, we report in Table 4 the values obtained from the HYDRA and Calar Alto spectra. The M3 type stars TTS 050646.1-031922 and TTS 050845.1-031653 have noisy spectra and we could not determine reliable $W(\mathrm{Li})$ values. The high value of $W(\mathrm{Li})$ for the M0-type star RX J0506.8-0318 may be due to line blending caused by possible multiplicity.

Seven of the 31 late type stars do show $\mathrm{H} \alpha$ in absorption, but the line is filled-in with some emission. Unfortunately, no 
Table 5. Stellar parameters of the PMS stars in L1616.

\begin{tabular}{|c|c|c|c|c|c|c|c|c|}
\hline Star & eff & $A_{\mathrm{V}}$ & ${ }^{\circ}$ & $\begin{array}{c}\log L_{X}^{b} \\
{\left[\mathrm{erg} \mathrm{s}^{-1}\right]}\end{array}$ & $\begin{array}{r}\text { Age (PS) } \\
{[\mathrm{Myr}]} \\
\end{array}$ & $\begin{array}{r}\text { Age }(\mathrm{DM}) \\
{[\mathrm{Myr}]} \\
\end{array}$ & $\begin{array}{r}(\mathrm{PS}) \\
{\left[M_{\odot}\right]} \\
\end{array}$ & $\begin{array}{r}M(\mathrm{DM}) \\
{\left[M_{\odot}\right]} \\
\end{array}$ \\
\hline 1RXS J0459 & $3.732 \pm 0.013$ & $1.02 \pm 0.15$ & $0.83 \pm 0.04$ & $31.00 \pm 0.34$ & $2.50 \pm 0.50$ & $2.50 \pm 0.60$ & $1.90 \pm 0.20$ & $2.25 \pm 0.20$ \\
\hline 1RXS J050416.9-0 & $3.671 \pm 0.014$ & $0.45 \pm 0.25$ & $0.40 \pm 0.03$ & $30.99 \pm 0.32$ & $2.10 \pm 0.90$ & $1.45 \pm 0.60$ & $1.65 \pm 0.10$ & $1.35 \pm 0.20$ \\
\hline RX J0506.6-0337 & $3.671 \pm 0.014$ & $0.00+0.25$ & $0.29 \pm 0.02$ & $30.57 \pm 0.14$ & 0.90 & 2.0 & 0.10 & .20 \\
\hline TTS 050646.1-03 & $3.532 \pm 0.015$ & $0.66 \pm 0.60$ & $-0.79 \pm 0.12$ & - & $2.10 \pm 1.00$ & $2.00 \pm 0.80$ & $0.25 \pm 0.07$ & $0.28 \pm 0.06$ \\
\hline КА JO & $3.584 \pm 0.020$ & $0.00+0.05$ & $-0.26 \pm 0.01$ & $29.96 \pm 0.21$ & $2.20 \pm 1.00$ & $1.50 \pm 0.52$ & $0.58 \pm 0.15$ & 1.0.0. \\
\hline RX J0506.8-0327 & $3.517 \pm 0.016$ & $0.21 \pm 0.52$ & $-0.24 \pm 0.10$ & $30.36 \pm 0.17$ & $0.30 \pm 0.20$ & $0.20 \pm 0.15$ & $0.19 \pm 0.06$ & $0.25 \pm 0.05$ \\
\hline RX J0506.9-031 & $3.631 \pm 0.020$ & $0.82 \pm 0.21$ & $0.20 \pm 0.05$ & $<30.50 \pm 0.14$ & $1.80 \pm 0.90$ & $0.85 \pm 0.25$ & $1.05 \pm 0.20$ & $0.82 \pm 0.25$ \\
\hline RX J0506.9-0319 NW & $3.547 \pm 0.018$ & $0.66 \pm 0.43$ & $-0.55 \pm 0.09$ & $<30.50 \pm 0.14$ & $1.90 \pm 1.00$ & $1.60 \pm 0.60$ & $0.35 \pm 0.09$ & $0.37 \pm 0.09$ \\
\hline RX J & $3.671 \pm 0.014$ & $3.28 \pm 0.33$ & $0.81 \pm 0.08$ & $<30.80 \pm 0.17$ & $0.30 \pm 0.20$ & $0.49 \pm 0.25$ & $1.90 \pm 0.30$ & 1.00 \\
\hline RX J0506.9-0320E & $3.644 \pm 0.013$ & $3.03 \pm 0.26$ & $0.57 \pm 0.06$ & $<30.80 \pm 0.17$ & $0.50 \pm 0.20$ & $0.45 \pm 0.20$ & $1.28 \pm 0.20$ & $0.97 \pm 0.15$ \\
\hline RX J0507.0 & $3.564 \pm 0.019$ & $0.81 \pm 0.27$ & $0.02 \pm 0.06$ & $30.27 \pm 0.18$ & $0.50 \pm 0.30$ & $0.45 \pm 0.20$ & $0.43 \pm 0.11$ & $0.39 \pm 0.07$ \\
\hline R & $54+0$ & 27 & $.39 \pm 0.06$ & 3 & $1.80 \pm 0.80$ & 0.60 & 0 & 0 \\
\hline $\mathrm{RXJ}$ & $3.618 \pm 0.023$ & $0.00+0.25$ & $-0.10 \pm 0.05$ & $30.26 \pm 0.14$ & $3.01 \pm 1.50$ & $1.95 \pm 0.90$ & $0.86 \pm$ & 0.7 \\
\hline TTS 050717.9-0324 & $3.547 \pm 0.016$ & $0.53 \pm 0.43$ & $-0.67 \pm 0.09$ & - & $2.30 \pm 0.90$ & $2.00 \pm 0.80$ & $0.35 \pm 0.09$ & $0.36 \pm 0.07$ \\
\hline RX J0507.4-0320 & $3.501 \pm 0.017$ & $0.00+0.19$ & $-0.53 \pm 0.04$ & $29.86 \pm 0.27$ & $0.50 \pm 0.30$ & $0.30 \pm 0.20$ & $0.15 \pm 0.05$ & $0.19 \pm 0.04$ \\
\hline TTs & 3.5 & $2.55 \pm 0.05$ & $<-0.29$ & - & $>0.60$ & $>0.70$ & 08 & 0 . \\
\hline TTS 050801.9-031732 & $3.532 \pm 0.015$ & $1.32 \pm 0.05$ & $-0.38 \pm 0.13$ & - & $0.75 \pm 0.30$ & $0.80 \pm 0.50$ & $0.26 \pm 0.08$ & $0.30 \pm 0.05$ \\
\hline TTS 050804.0-034052 & $3.517 \pm 0.016$ & $0.49 \pm 0.16$ & $-0.86 \pm 0.17$ & - & $1.80 \pm 1.00$ & $1.80 \pm 0.90$ & $0.20 \pm 0.06$ & $0.21 \pm 0.05$ \\
\hline TTS & 017 & $1.83 \pm 0.43$ & $<-0.45$ & - & $>0.40$ & $>0.20$ & $0.15 \pm 0.05$ & \pm 0.05 \\
\hline TTS $050845.1-031653$ & $3.532 \pm 0.015$ & $0.44 \pm 0.05$ & $-0.47 \pm 0.13$ & - & $0.95 \pm 0.60$ & $1.00 \pm 0.50$ & $0.27 \pm 0.08$ & $0.29 \pm 0.05$ \\
\hline 1RXS J051011.5-02 & $3.698 \pm 0.014$ & $0.21 \pm 0.23$ & $0.37 \pm 0.06$ & $31.05 \pm 0.29$ & $4.80 \pm 1.30$ & $3.40 \pm 1.40$ & $1.52 \pm 0.10$ & $1.65 \pm 0.06$ \\
\hline RX J0510.3-0330 ${ }^{a}$ & $3.725 \pm 0.011$ & $0.00+0.01$ & $0.64 \pm 0.04$ & $31.03 \pm 0.22$ & $2.50 \pm 0.50$ & $2.50 \pm 0.60$ & & $2.25 \pm 0.15$ \\
\hline 1RXS J05104 & $3.745 \pm 0.012$ & $0.38 \pm 0.14$ & $0.78 \pm 0.03$ & $31.04 \pm 0.34$ & $3.02 \pm 0.90$ & $4.00 \pm 1.00$ & $1.75 \pm 0.10$ & $2.00 \pm 0.25$ \\
\hline $\mathrm{LkH} \alpha 333$ & $3.631 \pm 0.020$ & $0.82 \pm 0.21$ & $0.26 \pm 0.05$ & $30.08 \pm 0.27$ & $1.20 \pm 0.80$ & $0.70 \pm 0.25$ & $1.05 \pm 0.20$ & $0.81 \pm 0.25$ \\
\hline 0974-1 & $3.644 \pm 0.013$ & 00 & $>0.40$ & - & $<1.00$ & $<0.68$ & $1.28 \pm 0.23$ & $0.95 \pm$ \\
\hline Kiso & $3.671 \pm 0.014$ & 0.00 & $>-0.18$ & - & $<12.00$ & $<10.02$ & $1.13 \pm 0.05$ & $1.12 \pm 0.03$ \\
\hline RX J0509.0-0315 ${ }^{a}$ & $3.698 \pm 0.013$ & $0.00 \pm 0.25$ & $0.67 \pm 0.04$ & $31.08 \pm 0.13$ & $2.00 \pm 0.90$ & $1.50 \pm 0.70$ & $2.00 \pm 0.10$ & $1.95 \pm 0.20$ \\
\hline RX J0512.3-0255 ${ }^{a}$ & $3.698 \pm 0.013$ & $0.06 \pm 0.16$ & $0.36 \pm 0.04$ & $31.09 \pm 0.15$ & $4.50 \pm 1.30$ & $3.50 \pm 1.40$ & $1.55 \pm 0.10$ & $1.64 \pm 0.06$ \\
\hline HD 293815 & $4.023 \pm 0.013$ & $0.77 \pm 0.25$ & $1.70 \pm 0.06$ & - & $3.00 \pm 0.90$ & $3.50 \pm 0.80$ & $2.70 \pm 0.10$ & $2.70 \pm 0.15$ \\
\hline RX J0510.1-0427 ${ }^{a}$ & $3.657 \pm 0.013$ & $0.25 \pm 0.20$ & $0.77 \pm 0.02$ & $31.26 \pm 0.12$ & $0.30 \pm 0.10$ & $0.35 \pm 0.10$ & $1.55 \pm 0.30$ & $1.21 \pm 0.25$ \\
\hline RX J0511.7-0348 & $3.698 \pm 0$ & $0.01+0.25$ & $0.51 \pm 0$ & $30.85 \pm 0.33$ & $3.00 \pm 1$ & $2.20 \pm 0.90$ & 1.80 & 1.80 \\
\hline L1616 MIR4 & $3.482 \pm 0.020$ & 0.00 & $>-0.56$ & - & $<0.40$ & $<0.10$ & $0.09 \pm 0.06$ & $0.15 \pm 0.04$ \\
\hline
\end{tabular}

${ }^{a}$ X-ray luminosity taken from Alcalá et al. (2000a); ${ }^{b}$ the quoted errors in the X-ray luminosities are those coming from the errors in the X-ray count-rates.

spectral information other than the detection of the $\mathrm{H} \alpha$ line or MIR emission is available for the stars Kiso A-0974-15, Kiso A-0974-17 and L1616 MIR4. However, their magnitudes and colors are typical of Orion PMS stars.

We assigned the classical (CTTS) or weak (WTTS) T Tauri star classification to the PMS stars (indicated in Table 4 with $\mathrm{C}$ or $\mathrm{W}$ respectively) assuming $W(\mathrm{H} \alpha)=-10 \AA$ as a conventional dividing line between these two types of objects. According to this convention, we find that our sample contains about five times more WTTS than CTTS. However, some stars with $W(\mathrm{H} \alpha)>-10 \AA$ do show a relatively strong infrared excess (see Sect. 3.5 below), which is a signature of residual circumstellar matter.

Despite the different spectral resolutions used, we can conclude that, for the weak $\mathrm{H} \alpha$ emission stars $(W(\mathrm{H} \alpha)>$ $-10 \AA)$, the strength of the $\mathrm{H} \alpha$ line is more or less constant, while for some stars with strong $\mathrm{H} \alpha$ emission $(W(\mathrm{H} \alpha)<-10 \AA)$, the line is highly variable: for instance, for the star RXJ0507.1-0321 we measure $W_{\text {СтіО }}(\mathrm{H} \alpha)=-72.9 \AA$ from the low-resolution spectrum, while from the intermediate-resolution spectrum we obtain $W_{\text {HYDRA }}(\mathrm{H} \alpha)=-15.50 \AA$; similarly, for the star $\mathrm{LkH} \alpha 333$ 
we measure $W_{\text {CтIO }}(\mathrm{H} \alpha)=-50.8 \AA$ from the low-resolution spectrum, while from the intermediate-resolution we obtain $W_{\text {HYDRA }}(\mathrm{H} \alpha)=-62.00 \AA$.

Lithium abundances, in the usual scale $\log H=12$, were derived from the $W(\mathrm{Li})$ and $T_{\text {eff }}$ values reported in Tables 4 and 5 respectively, using the non-LTE curves of growth given by Pavlenko \& Magazzù (1996), assuming $\log g=4.5$. The main source of error on the derived $\log N(\mathrm{Li})$ values is the uncertainty in the effective temperature. The estimated mean uncertainties on $T_{\text {eff }}$ are on the order of $\Delta T_{\text {eff }} \approx 150 \mathrm{~K}$. Taking this and a mean error of about $30 \mathrm{~m} \AA$ in $W(\mathrm{Li})$ into account, we estimate a mean error on the order of 0.2 to 0.25 dex in $\log N(\mathrm{Li})$. However, the assumption of $\log g=4.5$ affects significantly the lithium abundance determination, in the sense that a lower surface gravity would yield a higher lithium abundance. In particular, for stars with $\log T_{\text {eff }}$ less than about $3.7(\approx 5000 \mathrm{~K})$ and $\log W(\mathrm{Li})$ greater than about $2.5(\approx 320 \mathrm{~m} \AA)$, the difference in $\log N(\mathrm{Li})$ may rise to $0.3 \mathrm{dex}$, when assuming $\log g=3.5$. Hence, assuming $\log g=3.5$ would result in higher lithium abundances than when assuming $\log g=4.5$. Thus we adopt the most conservative value, $\log g=4.5$, which might lead to an underestimation of the abundance.

The $\mathrm{H} \alpha$ and lithium equivalent widths $W(\mathrm{Li})$, as well as the lithium abundances are reported in Table 4.

\subsection{Spectral energy distributions}

For the eighteen stars with optical and NIR photometry the spectral energy distributions (SEDs) were derived by computing the absolute fluxes at each wavelength from the dereddened magnitudes using the absolute fluxes for a zero magnitude star. The magnitudes were dereddened using intrinsic colors, derived from the spectral types via the spectral type versus intrinsic color relations for dwarfs by Bessell \& Brett (1988). For this purpose, the 2MASS data were homogenized to the Bessell \& Brett system using the transformation equations by Carpenter (2001).

For the stars Kiso A-0974-15, Kiso A-0974-17 and L1616 MIR4 we cannot deredden the observed magnitudes in this way because, as mentioned above, their spectral type could not be determined. We attempted, however, to derive their SEDs assuming no reddening. A major consequence of this assumption is that the luminosity of these stars will be underestimated. Therefore, we will consider their luminosities as lower limits only.

The SEDs are shown in Fig. 4. The SEDs of standard stars of the same spectral type as the PMS stars, but adjusted to the $R$-band flux of the corresponding PMS star, are also overplotted. In the case of the stars Kiso A-0974-15, Kiso A-097417 and L1616 MIR4 we overplot the SEDs of the standard stars which minimize the residuals relative to the observed fluxes at each optical wavelength. The spectral type of the standards which better match the observed optical SEDs are K5, K3 and M6 for Kiso A-0974-15, Kiso A-0974-17 and L1616 MIR4 respectively. Interestingly, Kiso A-0974-15 and L1616 MIR4 show a detectable infrared excess, while the SED of Kiso A-0974-17 follows very closely the SED of a K3 type star also in the NIR.

Seven stars in our sample show infrared excess, and three of them (RX J0506.9-0320W, RX J0506.9-0320E and RX J0512.3-0255) show weak $\mathrm{H} \alpha$ emission. As mentioned in the previous section, the $\mathrm{H} \alpha$ emission line may be strongly variable. Evidence of such variability in T Tauri stars with strong infrared excess has been detected in the past (cf. Alcalá et al. 1993).

From the point of view of the infrared emission and following the classification of young stellar objects by Lada (1987), we conclude that there are a factor of about three more class-III infrared sources than class-II infrared sources.

\subsection{Stellar luminosities}

The luminosities were obtained in three different ways, assuming a distance of $450 \mathrm{pc}$ (i.e. the Orion distance) and a normal interstellar extinction law: 1) For the stars with optical and NIR photometry we integrate the SEDs following the methods described in Alcalá et al. (1997). We designate this luminosity as $L_{\mathrm{SED}}$; 2) For the stars with optical photometry, the $I_{\mathrm{C}}$ magnitude corrected for interstellar extinction was used to obtain the bolometric luminosity $\left(L_{\mathrm{OPT}}\right)$ by adopting a bolometric correction appropriate to a main-sequence star of the same spectral type. The relations given by Bessell \& Wood (1984) were used to compute the bolometric corrections; 3) For the stars with NIR photometry only, the $K_{\mathrm{S}}$ magnitudes corrected for IS extinction were used to estimate luminosities $\left(L_{\mathrm{IR}}\right)$ of the program stars. For the stars Kiso A-0974-15, Kiso A-0974-17 and L1616 MIR4, we use the spectral types K5, K3 and M6 respectively, estimated from their SEDs as explained above, in order to derive the bolometric corrections.

In general $L_{\mathrm{SED}}$ is in agreement with both $L_{\mathrm{OPT}}$ and $L_{\mathrm{IR}}$ within less than 0.15 dex. The latter is not true, however, for stars with strong infrared excess, in which the disk contribution may yield a difference as high as 0.69 dex; an example is TTS 050646.1-031922 which shows a rising SED in the NIR and is one of the strongest $\mathrm{H} \alpha$ emitters in our sample.

The luminosities $L_{\mathrm{SED}}$ include the non-stellar disk contribution, while those derived using bolometric corrections in the optical, are closer to the real stellar luminosities. Therefore, for the rest of the paper we use preferentially the luminosities $L_{\mathrm{OPT}}$ or $L_{\mathrm{IR}}$, derived from the available optical or NIR data, in that order.

For the stars with NIR photometry only, the luminosity may be overestimated when there is infrared excess, like in the case of TTS 050801.4-032255 and TTS 050836.6-030341. Hence, we consider $L_{\mathrm{IR}}$ as an upper limit for these two stars. The rest of the stars with NIR photometry only have little evidence of strong infrared excess, as can be seen from the NIR color-color diagram in Fig. 3 . Hence, their $L_{\mathrm{IR}}$ luminosities are reliable.

Since the star HD 293815 is saturated in our WFI images, we use the photometry from the literature (Neckel \& Chini 1980) in order to determine its luminosity: using the magnitudes reported in Table 3 and the spectral type B9V, we find $A_{\mathrm{V}}=0.77$ assuming a normal extinction law. We then obtain 

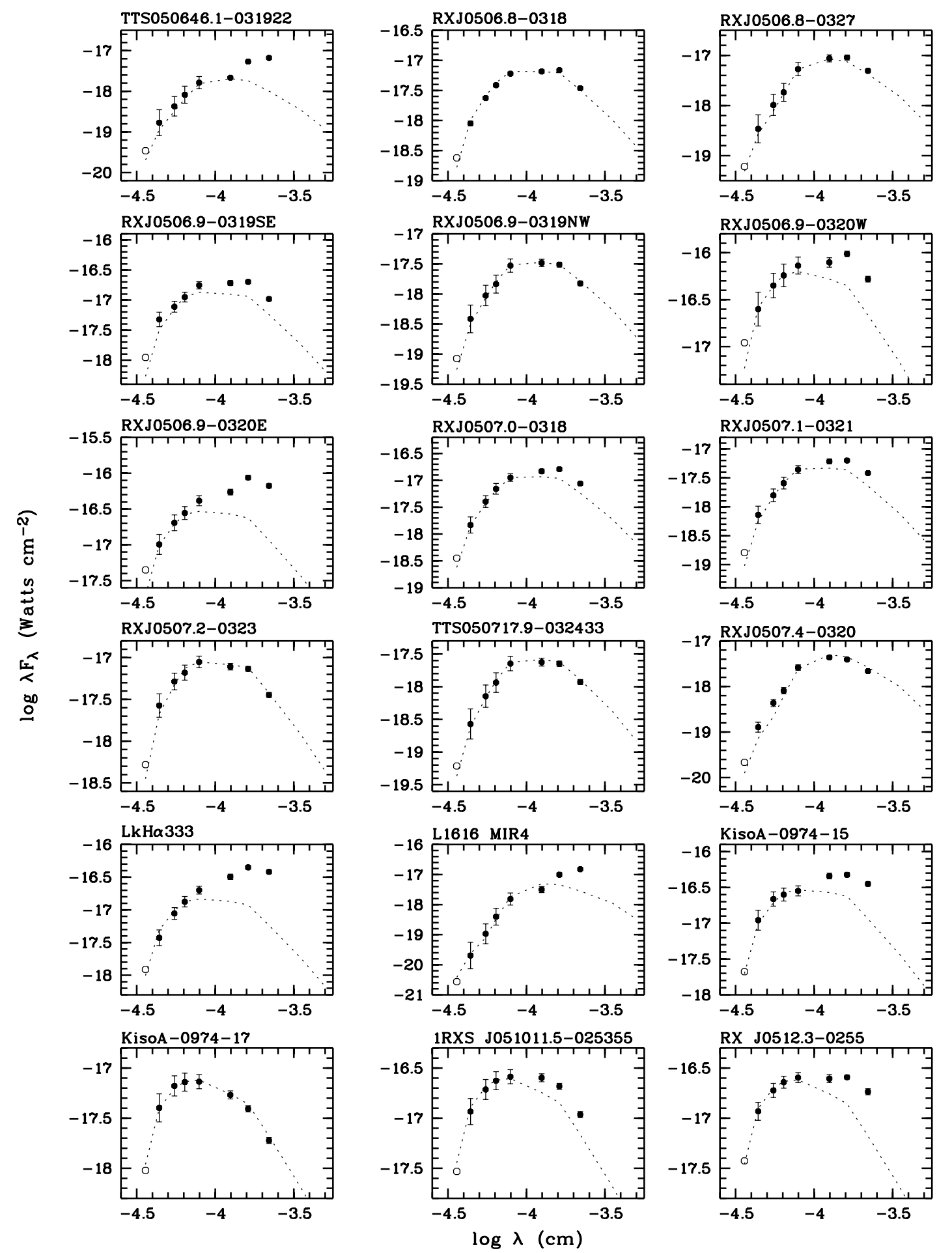

Fig. 4. Spectral energy distributions (SEDs) of PMS stars in L1616 (dots). The SEDs of standard stars of the same spectral type as the PMS stars are also overplotted (dotted lines). The open circle in each SED represents the $U$ band flux for a star of the same spectral type as the corresponding PMS star. The error in each band mainly reflects the uncertainty of the interstellar extinction due to the uncertainty in spectral type.

an absolute magnitude $M_{\mathrm{V}}=1.01$, adopting a distance of $450 \mathrm{pc}$. Using the bolometric correction corresponding to spectral type B9V, we obtain an absolute magnitude $M_{\mathrm{BOL}}=0.50$, and hence, a luminosity $\log L / L_{\odot}=1.70$.
The resulting values for the interstellar extinction, $A_{\mathrm{V}}$, and the derived stellar luminosities are reported in Table 5. The errors in $A_{\mathrm{V}}$ are mainly due to the uncertainty in spectral type. 


\subsection{X-ray luminosities}

The X-ray luminosities were derived using the count rates reported in Table 1 (assuming isotropic radiation) using the expression $L_{\mathrm{X}}=4 \cdot \pi \cdot d^{2} \cdot f_{\mathrm{X}}$, where $d$ is the distance and $f_{\mathrm{X}}$ is the $\mathrm{X}$-ray flux at the earth in $\mathrm{erg} \mathrm{s}^{-1} \mathrm{~cm}^{-2}$. The latter is given by $f_{\mathrm{X}}=E C F \cdot Z$, where $E C F$ is the energy conversion factor and $Z$ is the broad-band $X$-ray count rate.

For the HRI X-ray sources, we adopt the ECF values from Huensch et al. (1990) $)^{5}$, assuming a $1 \mathrm{keV}$ Raymond-Smith spectrum. The values for the hydrogen column density $\log N_{\mathrm{H}}$ were determined using the interstellar extinction values reported in Table 5 and the relation $N_{\mathrm{H}} \mathrm{cm}^{-2}=1.763 \times 10^{21} \cdot A_{\mathrm{V}} \mathrm{mag}^{-1}$ by Paresce (1984) and Vrba \& Rydgren (1985).

For the other X-ray sources we adopt the average value $E C F=1.2 \times 10^{-11} \mathrm{erg} \mathrm{cm}^{-2}$ count $^{-1}$, consistent with the one derived for X-ray emitting PMS stars observed with the ROSAT-PSPC (e.g. Alcalá et al. 2000b).

The X-ray luminosities for the X-ray detected PMS stars in L1616 are reported in Table 5. The X-ray luminosities for the previously known RASS PMS stars were taken from Alcalá et al. (2000a).

Since the stars RX J0506.9 - 0319 SE and RX J0506.9 0319 NW fall inside the same X-ray error circle, we consider their X-ray luminosity as an upper limit. The latter also applies to RX J0506.9-0320W and RX J0506.9-0320E.

\subsection{On the $X$-ray emission of the PMS stars in L1616}

The X-ray detected PMS stars in L1616 have X-ray luminosities in the range $\log L_{X}=29.86$ to $\log L_{X}=31.26$, which is well within the X-ray luminosity range of PMS stars in the Orion Nebula detected in deep ROSAT (Gagné et al. 1995) and Chandra (Flaccomio et al. 2003) observations.

As also found in the Orion Nebula, in L1616 there are PMS stars with rather high X-ray luminosities as compared to the X-ray luminosities of PMS stars in T associations like Chamaeleon or Lupus. However, this is explained in terms of the extreme youth of the Orion stars.

Despite the large errors and the small sample, we find a number of correlations between the X-ray luminosity and the stellar parameters of the PMS stars in L1616. The X-ray luminosity is correlated with the spectral type, in the sense that the late type stars possess a lower level of X-ray emission than the early type stars; the X-ray luminosity is also correlated with the bolometric luminosity. These two correlations may simply reflect the fact that the X-ray luminosity is correlated with the stellar mass. This correlation has indeed been found in previous investigations by Walter et al. (1996) in the ScoCen association, by Feigelson et al. (1993) in the Chamaeleon I SFR and more recently also in the Orion Nebula by Flaccomio et al. (2003).

In Fig. 5 we show the scatter plot of X-ray luminosity versus the mass determined from the D'Antona \& Mazzitelli (1997)

\footnotetext{
${ }^{5}$ See also http://hea-www.harvard.edu/rosat/rsdc_www/ hricalrep.html
}

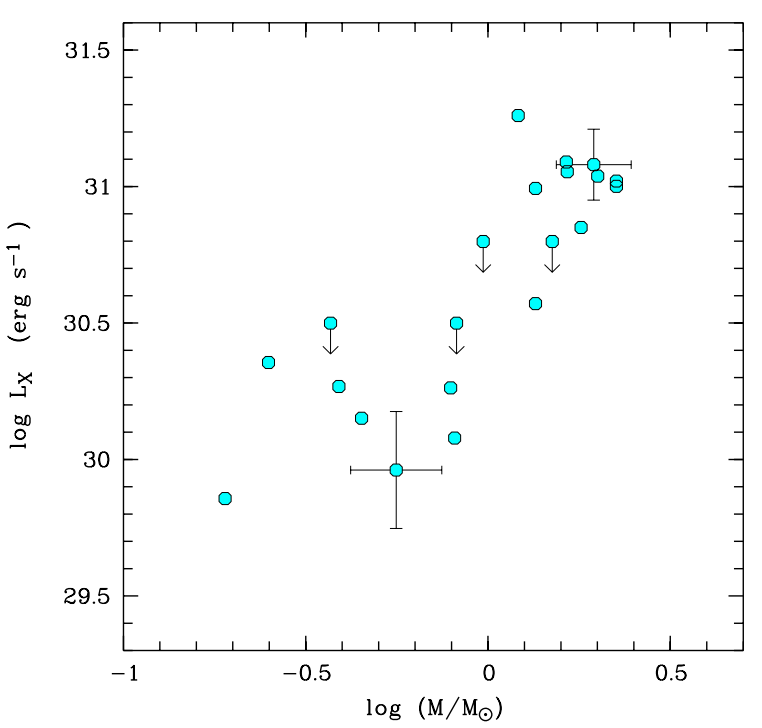

Fig. 5. Scatter plot $\log L_{\mathrm{X}}$ versus $\log M / M_{\odot}$ for the stars in our sample. The stars for which $\log L_{\mathrm{X}}$ is considered as an upper limit are indicated with low-arrow symbols. Errors are indicated for two stars, representing the full range of errors.

PMS tracks. Although the errors are relatively large, there is a clear correlation between the two quantities.

Since the sample is small, we do not attempt to perform a fit to the data. However, we note that, despite the differences in the instrumental set-ups between ROSAT and Chandra, the trend observed in Fig. 5 is fairly consistent with the one in Figs. 4 and 7 by Flaccomio et al. (2003). The results do not change if we use masses derived from the Palla \& Stahler (1999) PMS tracks.

Within the mass range of our sample $\left(0.15<M / M_{\odot}<2.5\right)$, the values of $\log L_{\mathrm{X}} / L_{\mathrm{bol}}$ are, within the errors, quite constant with mass. Most of the stars are very close, but below the saturation limit $\log L_{\mathrm{X}} / L_{\mathrm{bol}}=-3$. We find an average value $\left\langle\log L_{\mathrm{X}} / L_{\mathrm{bol}}\right\rangle=-3.2 \pm 0.3$ for the stars in our sample which, again, is consistent with the value found for many PMS stars in the Orion Nebula (Gagné et al. 1995; Flaccomio et al. 2003).

\subsection{Completeness}

Our X-ray and HYDRA observations are spatially unbiased in an area of one square degree in the head of the cloud. However, the RASS observations have a different sensitivity limit relative to the ROSAT HRI observations.

\subsubsection{X-ray completeness}

We estimate that the sensitivity limit of the ROSAT HRI observations is on the order of $\log L_{\mathrm{X}}=29.9$. PMS stars at $450 \mathrm{pc}$ with X-ray luminosities below this limit escaped detection in our HRI X-ray observations. Using this limit and the fact that practically all the PMS stars in L1616 have values of X-ray to bolometric luminosity $\log L_{\mathrm{X}} / L_{\mathrm{bol}}>-3.5$, we estimate a limiting bolometric luminosity $\log L / L_{\odot}=-0.2$.

The RASS sensitivity limit is about 0.01 count $\mathrm{s}^{-1}$ which means a limiting X-ray luminosity of about $\log L_{X}=30.5$ 
Table 6. Completeness of the unbiased lithium survey as function of the $R_{\mathrm{USNO}}$ magnitude range.

\begin{tabular}{crrr}
\hline \hline$R_{\text {USNO mag. range }}$ & $\begin{array}{r}\text { No. } \\
\text { stars }\end{array}$ & $\begin{array}{r}\text { No. } \\
\text { observed }\end{array}$ & $\%$ \\
\hline $11-12$ & 38 & 21 & 55 \\
$12-13$ & 68 & 43 & 60 \\
$13-14$ & 137 & 69 & 50 \\
$14-15$ & 254 & 66 & 26 \\
$15-16$ & 575 & 224 & 39 \\
\hline
\end{tabular}

(using a counts to energy conversion factor of $1.2 \times$ $10^{-11} \mathrm{erg} \mathrm{cm}^{-2}$ count $^{-1}$ ), at $450 \mathrm{pc}$. This yields a limiting bolometric luminosity on the order of $\log L / L_{\odot}=0.4$.

Of the 22 new PMS stars in our survey, 15 detected in $\mathrm{X}$-rays, while all 22 were observed in the optical spectroscopic observations. Therefore, the X-ray sample is about $70 \%$ complete relative to the spectroscopic sample.

\subsubsection{Optical completeness}

In order to determine the optical completeness, we assume that the USNO catalog is complete down to the magnitude limit $R_{\text {lim }}=16.0$ of our HYDRA observations.

In Table 6 the fraction of stars observed with HYDRA relative to the sample extracted from the USNO catalog in the L1616 area is given as a function of magnitude range. On average, we completed about $40 \%$ of the USNO sample, but the percentage of observed objects is quite different from a magnitude range to the other.

Our sensitivity limit $R_{\lim }=16.0$ can be translated into a luminosity limit using the $\left(V-R_{\mathrm{C}}\right)$ colors and bolometric corrections $\left(B C_{\mathrm{V}}\right)$ reported in Hartigan et al. (1994) by means of the expression $\log L / L_{\odot}=0.4\left(12.94-B C_{\mathrm{V}}-(V-R)-R_{\lim }\right)$, in which the distance modulus of 8.3 has been adopted. For an M 2 type star this $\operatorname{limit}$ is $\log L / L_{\odot}=-0.98$, while for an M 6 type star it is $\log L / L_{\odot}=-0.22$.

\section{Discussion}

The previous investigations provided some evidence for a recent and ongoing star formation event in L1616. Star formation in this region may have been triggered by the Orion OB stars (Ramesh 1995; Stanke et al. 2002), in which case sequential star formation should be observed.

Our multi-wavelength observations demonstrate that there is indeed a low-mass PMS population in the region. Thus, we can use these stars as tracers for a possible sequence of star formation events but, before doing that, we need first to assess the low-mass PMS nature of the stars we have found in the region, as well as their association with Orion.

\subsection{On the PMS nature of the lithium stars in L1616}

The presence of strong lithium absorption in the spectra of stars later than about G5 is the main criterion for the PMS

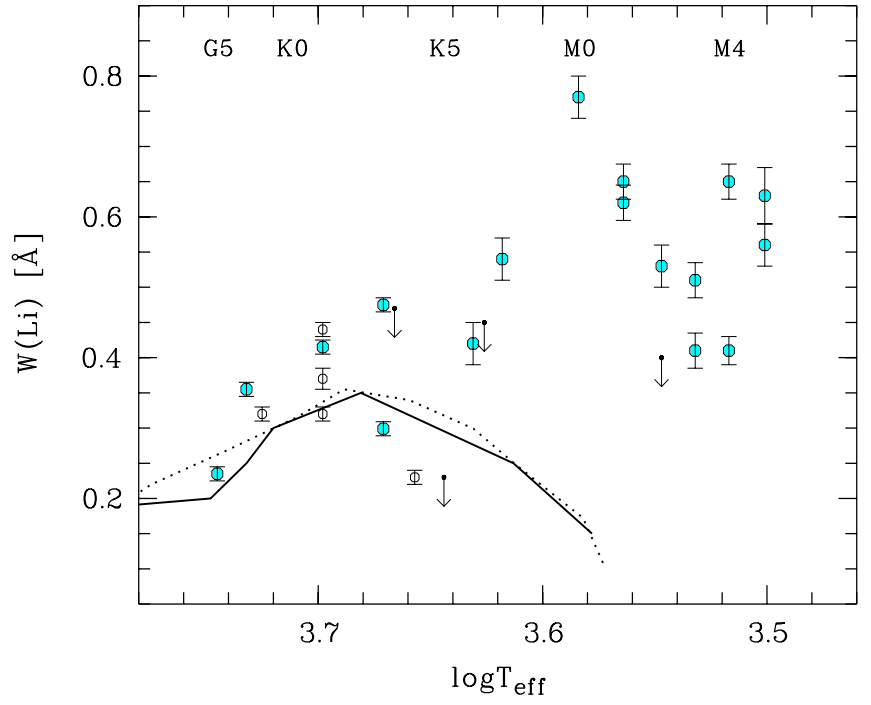

Fig. 6. Lithium equivalent width versus effective temperature for the stars in L1616. The thick and dotted lines represent the upper envelope for young open clusters as adopted by Martín \& Magazzù (1999) and Preibisch et al. (1998) respectively. The previously known RASS PMS stars are represented with open circles, while the stars RX J0506.9-0319 SE, RX J0506.9-0319 NW, RX J0506.9-0320W and RXJ0506.9-0320E are plotted as upper limits (see text for details). For the sake of clarity the $\log T_{\text {eff }}$ values of the stars RX J0506.9-0319 SE and RX J0506.9-0320W have been shifted by -0.005 dex.

assessment, because the element is efficiently destroyed by convective mixing in the stellar interiors when the temperature at the bottom of the convective layer reaches about $2 \times 10^{6} \mathrm{~K}$ (Bodenheimer 1965; D’Antona \& Mazzitelli 1994). The $W(\mathrm{Li})$ versus $T_{\text {eff }}$ diagram has been adopted by many authors as a standard tool to check whether or not the lithium strength is consistent with a low-mass PMS nature (Magazzù et al. 1997; Covino et al. 1997; Wichmann et al. 1999; Alcalá et al. 2000a; Mamajek et al. 2002).

In Fig. 6 the $W(\mathrm{Li})$ versus $T_{\text {eff }}$ diagram for the stars in our sample is shown. Despite the different resolutions used, it is evident that most of the stars in our sample have a stronger lithium absorption line than stars in young open clusters. The four stars RX J0506.9-0319 SE, RX J0506.9-0319 NW, RX J0506.9-0320W and RX J0506.9-0320E, are plotted as upper limits because their $W(\mathrm{Li})$ values have been measured from low-resolution spectra ${ }^{6}$ (see Sect. 3.4). Also, the comparison of the lithium abundance of the stars in our sample with that of stars in young clusters, like IC 2602 (see Fig. 7) shows that most of them should be as young as, or younger than $30 \mathrm{Myr}$.

A more precise estimate for the age of the stars in the L1616 SFR can be derived by comparing their position in the Hertzsprung-Russell (HR) diagram with theoretical PMS

\footnotetext{
6 The strength of the $\mathrm{Li}+\mathrm{Fe}$ lines blend in the low-resolution spectra of these stars appears stronger than the Ca I $\lambda 6718 \AA$ absorption line. When this happens for stars with spectral type later than about $\mathrm{K} 0$, the unblended $\mathrm{Li}$ absorption line is generally stronger than the Ca I absorption line, which provides a good evidence for a PMS nature (see Tripicchio et al. 2000).
} 


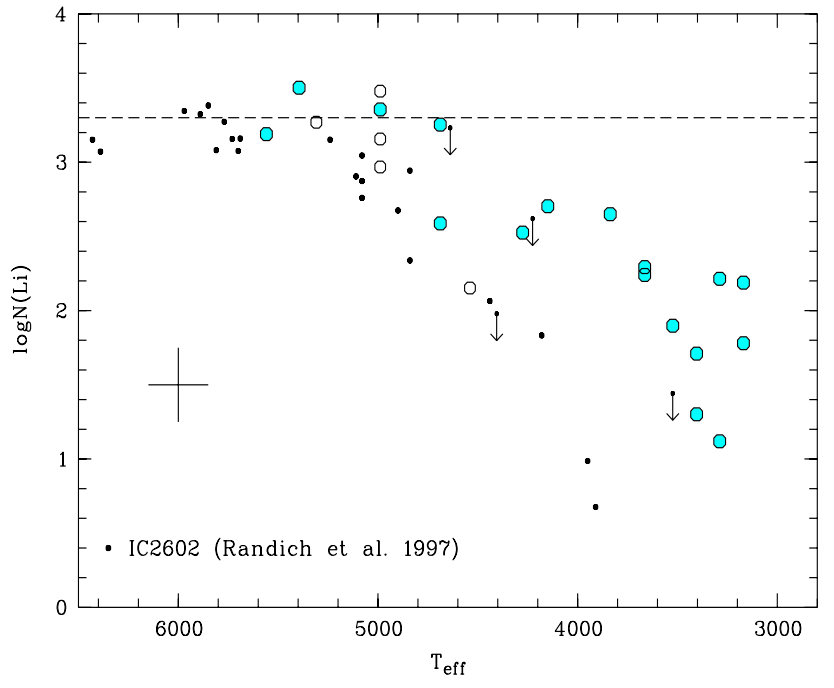

Fig. 7. Lithium abundance versus effective temperature for the PMS stars in L1616. The stars of the 30 Myr old cluster IC 2602 by Randich et al. (1997) are also plotted. The dashed line represents the cosmic lithium abundance of 3.3 in the $\log H=12$ scale. The estimated maximum errors in effective temperature and lithium abundance are also indicated. The symbols for the stars in our sample are as in Fig. 6. For the sake of clarity the $\log T_{\text {eff }}$ values of the stars RX J0506.9-0319 SE and RX J0506.9-0320W have been shifted by $-50 \mathrm{~K}$.

evolutionary tracks. Using the effective temperatures and luminosities reported in Table 5 we have determined masses and ages by comparison with the theoretical PMS evolutionary tracks by D' Antona \& Mazzitelli (1997) and by Palla \& Stahler (1999). The resulting interpolated values for the masses and ages are reported in Table $5(M(\mathrm{DM})$, Age(DM) and $M(\mathrm{PS})$, Age(PS) for D'Antona \& Mazzitelli 1997 and Palla \& Stahler 1999 respectively).

In Fig. 8 the HR diagram for the stars in our sample is shown. The PMS evolutionary tracks by PS99 are overplotted.

The average age for the stars in the L1616 region as derived from the DM97 and PS99 evolutionary tracks is $1.3 \mathrm{Myr}$ and 1.6 Myr respectively. Given the involved uncertainties and considering the upper and lower limits for the luminosity of some of the stars, we can safely conclude that the studied stars have an average age of 1-2 Myr, with a dispersion of about $1 \mathrm{Myr}$. This isochronal age is consistent with the PMS evolution time of a ZAMS star like HD 293815, whose position on the HR diagram is consistent with a ZAMS object when adopting the same distance of $450 \mathrm{pc}$ as for the other stars. This provides further support for the distance of L1616 SFR to be about $450 \mathrm{pc}$ and also for the membership of HD 293815 to the L1616 SFR.

Based on CO observations, Ramesh (1995) found an excess velocity of about $1.5 \mathrm{~km} \mathrm{~s}^{-1}$ above the virial equilibrium velocity of the cloud, which implies that its virial mass is about five times larger than the observed value. He concluded that the energy input of the stars in the cluster is fragmenting the cloud and, given its size of about $2 \mathrm{pc}$, the excess motions of the gas above the virial equilibrium suggest that the fragmentation may have lasted for the past 1-2 Myr. The latter is consistent with the age we determine for the stars in the region.

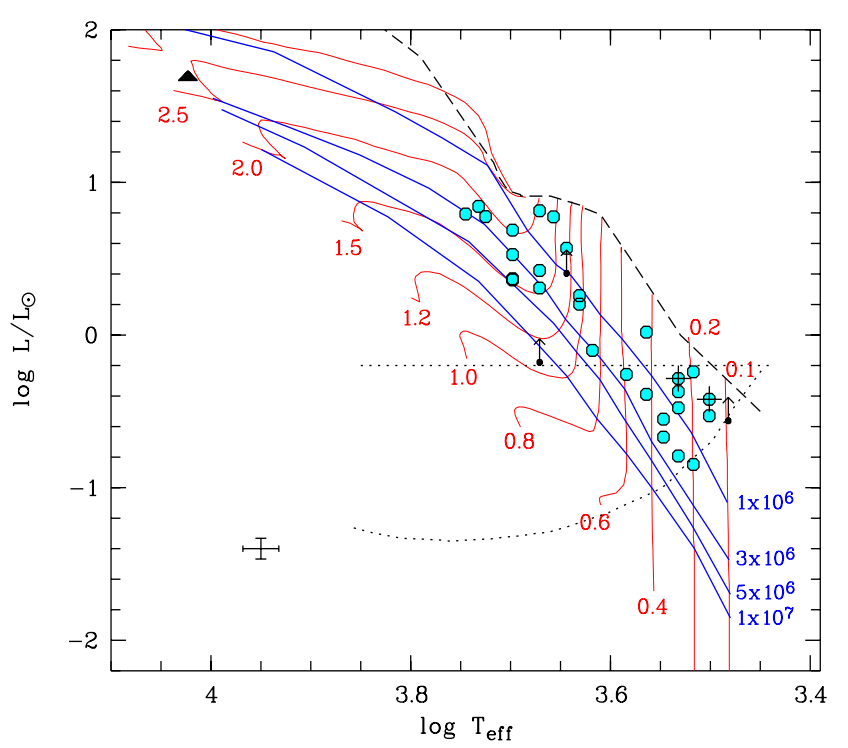

Fig. 8. HR diagram of the known PMS stars in L1616 SFR. The points with the up-arrow symbols represent the stars Kiso A-097415, Kiso A-0974-17 and L1616 MIR4 for which the luminosity may be underestimated (see Sect. 3.5). The stars with a "+" symbol overplotted are TTS 050801.4-032255 and TTS 050836.6-030341 for which the luminosity might be overestimated (see Sect. 3.6). The star HD 293815 is represented with a filled triangle. The theoretical PMS evolutionary tracks by Palla \& Stahler (1999) are overplotted. The dashed line represents the birth line. The average errors are indicated in the lower left. The horizontal and curved dotted lines represent the X-ray and optical sensitivity limits $\log L_{\mathrm{X}}=29.9$ and $R_{\lim }=16.0 \mathrm{mag}$ respectively.

Another important issue regards the kinematics of the stars. From the CO observations by Maddalena et al. (1986) an average radial velocity of $8.7 \pm 3.7 \mathrm{~km} \mathrm{~s}^{-1}$ in the Local Standard of Rest (LSR) is derived for the Orion clouds, which means an average heliocentric radial velocity of about $25 \mathrm{~km} \mathrm{~s}^{-1}$. The extensive survey by Dolan $\&$ Mathieu (2001) in the $\lambda$ Ori region showed that the average heliocentric radial velocity of the stars in that region is $24.5 \pm 2.3 \mathrm{~km} \mathrm{~s}^{-1}$. Based on the $W(\mathrm{Li}) \mathrm{vs}$. $R V$ diagram, Dolan \& Mathieu determined an empirical cut-off value of $W(\mathrm{Li})=200 \mathrm{~m} \AA$ as the dividing line between field stars and members of the $\lambda$ Ori region.

As can be seen from Fig. 9, all the stars in our sample have $W(\mathrm{Li})$ values above $200 \mathrm{~m} \AA$ and their heliocentric radial velocities are consistent with the average value of about $25 \mathrm{~km} \mathrm{~s}^{-1}$. Hence, despite the large angular distance relative to the Orion main molecular clouds and the $\lambda$ Ori region, the stars in L1616 possess strong lithium and radial velocities that are consistent with those of the Orion molecular clouds and $\lambda$ Ori PMS stars. Furthermore, L1616 corresponds to the CO clump No. 13 by Maddalena et al. (1986), for which a LSR radial velocity of $7.7 \mathrm{~km} \mathrm{~s}^{-1}$ is measured. The latter means a heliocentric radial velocity of about $22.6 \mathrm{~km} \mathrm{~s}^{-1}$, in perfect agreement with the average heliocentric radial velocity of the stars in our sample.

There seems to be a weak correlation between the $W(\mathrm{Li})$ values and the heliocentric radial velocities (e.g. Fig. 9) in the sense that higher $W(\mathrm{Li})$ values correspond to higher radial velocities. This might mean that (dominantly) our M-type stars, 

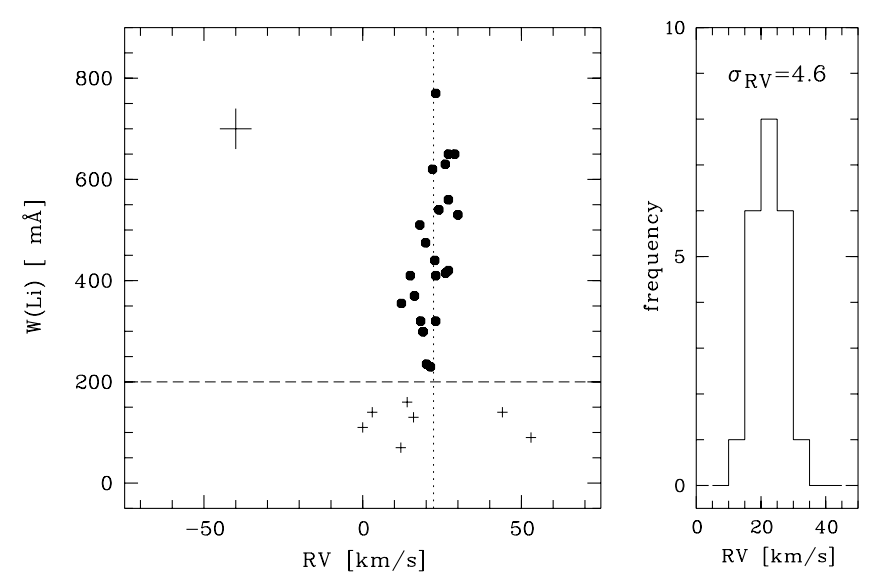

Fig. 9. Left panel: the lithium equivalent width vs. the heliocentric radial velocity of stars in L1616 (dots). The dashed line defines the $200 \mathrm{~m} \AA$ criterion by Dolan \& Mathieu (2001) for PMS stars in the $\lambda$ Ori region. The dotted line represents the average radial velocity $\langle R V\rangle=22.3 \pm 4.6 \mathrm{~km} \mathrm{~s}^{-1}$ for the strong lithium stars in L1616. The additional lithium stars reported in Appendix B are represented with crosses. For comparison purposes, we show the same radial velocity range as in Fig. 3 by Dolan \& Mathieu (2001). The average errors of $W(\mathrm{Li})$ and radial velocity measurements are indicated in the upper left; Right panel: the radial velocity distribution of stars in L1616. The standard deviation on the average radial velocity is indicated.

which are typically those with the higher $W(\mathrm{Li})$ values, have somewhat larger radial velocities compared to the early type stars. Given the large errors involved we consider this correlation as tentative only.

Notwithstanding the average error of $5 \mathrm{~km} \mathrm{~s}^{-1}$, the radial velocity distribution of the stars in L1616 shows a well defined peak at $22.3 \mathrm{~km} \mathrm{~s}^{-1}$ with the standard deviation of $4.6 \mathrm{~km} \mathrm{~s}^{-1}$ (see Fig. 9). The fact that the latter is consistent with the average $R V$ error, may indicate that the velocity dispersion of the stars must be less than $5 \mathrm{~km} \mathrm{~s}^{-1}$, although higher spectral resolution $(R>20000)$ than used here is necessary in order to determine the velocity dispersion of the stars in our sample.

Finally, the X-ray properties of the stars discussed here, are quite consistent with those of Orion low-mass PMS stars (see Sect. 3.8).

From the above discussion we conclude that all the latetype stars listed in Table 3 are coeval, low-mass PMS stars which, together with HD 293815, are members of the visible population of the L1616 cometary cloud in Orion.

\subsection{Triggered star formation in $L 1616$}

It is interesting to note that, without taking into account the upper and lower limits on the luminosities and despite the uncertainties, the age dispersion of the visible PMS stars in L1616 is quite small, about $1 \mathrm{Myr}$, regardless of the PMS evolutionary tracks used.

Similar results regarding the small age spread of PMS stars were found by Walter et al. (1994) and Preibisch \& Zinnecker (1999) in the low-mass PMS population in the Upper Scorpius OB Association. These authors interpret the small age spread in terms of efficient and quick star formation, triggered by the shock wave of a supernova explosion in the nearby Upper Centaurus-Lupus association.

Another important issue is the high star formation efficiency (SFE) in L1616. From CO observations, Ramesh (1995) determine a total mass of $180 M_{\odot}$ for the cloud in L1616. The total mass in PMS stars in L1616 is at least $30 M_{\odot}$. Therefore, the star formation efficiency (stellar mass/total mass) is about $14 \%$, which is in good agreement with the determination by Ramesh (1995) based on FIR fluxes. The SFE in L1616 is significantly high when compared with the average value of a few percent $(<3 \%)$, found in other nearby SFRs.

The spatial distribution of the PMS stars in L1616 is shown in Fig. 10. The size of the symbols is proportional to the stellar age determined from the DM97 tracks. It is evident that the vast majority of the PMS stars discussed here are distributed to the East of the head of the cometary cloud, while the class0 protostar, and the MIR sources are concentrated in the head of the cometary cloud. The Orion OB stars are some $8^{\circ}$ (about $60 \mathrm{pc}$ ) to the North-East.

Therefore, there is indeed evidence for sequential star formation, with the Orion OB stars probably being the sources that triggered the star formation in the region (Stanke et al. 2002).

Two scenarios for triggered star formation have been envisioned:

i) Ramesh (1995) proposed that the L1616 cloud, originally located about $4 \mathrm{pc}$ from the blue supergiant star $\epsilon$ Ori, was propelled due to the strong UV radiation field from the supergiant star: an ionization front was driven in the cloud, that generated a pressure gradient in the regions of the cloud facing the supergiant star, producing a recoil that accelerated the cloud away from the star. Hence, the cloud moved from its initial location to its present position on a time scale of about $5 \mathrm{Myr}$. From these considerations, as well as from CO observations, Ramesh (1995) determined the expected tangential velocity for the cloud to be about $12 \mathrm{~km} \mathrm{~s}^{-1}$.

The only certain way to confirm whether the cloud was indeed propelled by $\epsilon$ Ori is by means of proper motion measurements. Unfortunately, such measurements are not available for the PMS stars discussed here. However, the proper motion components $\mu_{x}=-4.40, \mu_{y}=-3.30$ of HD 293815 (which yield a proper motion velocity of $v_{\mathrm{T}}=11.8 \mathrm{~km} \mathrm{~s}^{-1}$, at the distance of $450 \mathrm{pc}$ ) would be consistent with the "rocket acceleration" hypothesis by Ramesh (1995).

In this scenario, the star formation starts while the cloud is moving away from the supergiant star. The average age of 1-2 Myr of the PMS stars in L1616 would imply that star formation in the cloud started some 3-4 Myr after the cloud was propelled from its original position and when it was at a distance of about 40 pc from $\epsilon$ Ori (see Fig. 8 by Ramesh 1995). Therefore, since the cloud is presently located at about $60 \mathrm{pc}$ from $\epsilon$ Ori, one should expect the PMS stars to be distributed up to some $20 \mathrm{pc}$ to the North-East of the present cloud position, i.e. some $2.5^{\circ}$ North-East of the cloud (assuming a distance of $450 \mathrm{pc})$.

As seen from Fig. 10 we find PMS stars only up to about $1.5^{\circ}$ to the North-East of the cloud, which means about $12 \mathrm{pc}$. However, this may be due to the limits of our surveyed area. There might be other low-mass PMS stars more than $2^{\circ}$ 


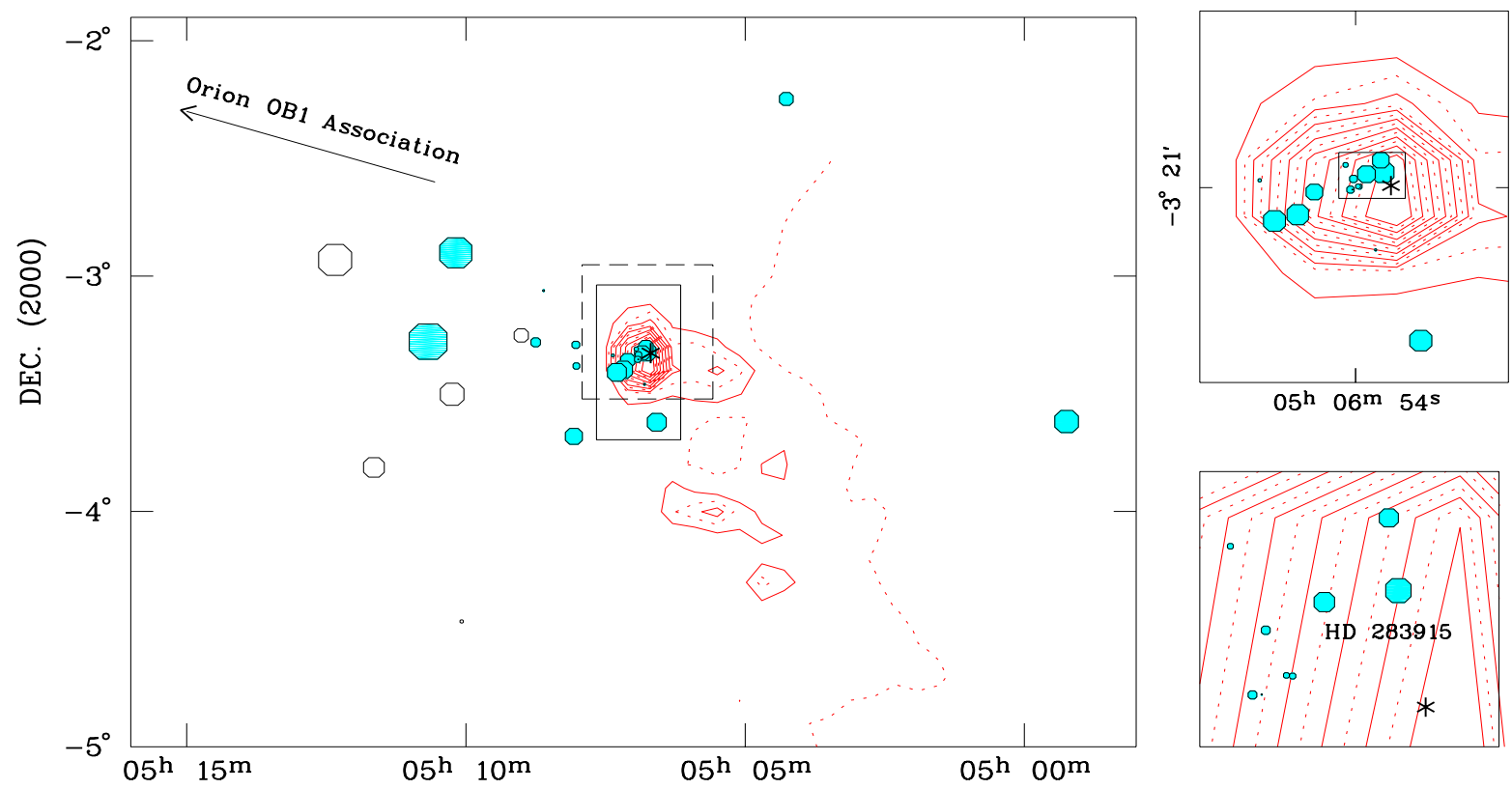

R.A. (2000)

Fig. 10. Spatial distribution of the PMS stars in L1616 (shaded and open octagons) overplotted on the $100 \mu$ m IRAS contour map of the region (shaded lines). The millimeter source by Stanke et al. (2002) is represented with an asterisk. The open octagons represent the five previously known RASS PMS stars by Alcalá et al. (2000a). The dashed square marks the area surveyed in the optical with the WFI and the rectangle indicates the zoomed area shown in the upper right panel. The small continuous rectangle in the upper right panel is further zoomed in the lower right panel. The size of the symbols is proportional to the stellar age determined from the DM97 tracks. The arrow shows the direction to the Orion OB1 association, at about $60 \mathrm{pc}$ to the North-East of L1616.

North-East from the cloud. In fact, when inspecting the Kiso $\mathrm{H} \alpha$ survey catalog, there are tens of $\mathrm{H} \alpha$ emission line stars further to the North-East of the area surveyed by us. It is, however, unclear whether these stars are related to the L1616 cloud or were formed from another much larger and now dispersed cloud, in the space between the Ori OB association and the present position of L1616. Photometry and spectroscopy of these stars would be desirable in order to determine their SEDs and kinematics, as well as to confirm their PMS nature using the lithium line and investigate whether they are coeval with the stars discussed here.

ii) Alternatively, the star formation may have been triggered by the impact of the Orion OB stars on a much larger cloud complex, between the Orion OB stars and the present location of L1616, from which the PMS stars that we are now seeing were formed. The larger cloud complex was subsequently dispersed by the impact of the newly formed stars.

Stanke et al. (see their Fig. 7) proposed that the oldest population may be represented by the PMS stars dispersed some 8-15 pc East of L1616, followed by the emission line stars found at the eastern edge of the cloud. The partially embedded sources and the millimeter and MIR sources would represent the most recent star formation episode.

As can be seen from Fig. 10, the PMS stars are distributed in a more or less continuous way up to about some $1.5^{\circ}$ $(\approx 12 \mathrm{pc})$ to the East of the cloud, but there is no clear evidence for a boundary between the on-cloud PMS stars and those distributed to the east. However, the infrared properties of the oncloud stars differ from those of the off-cloud stars: the infrared

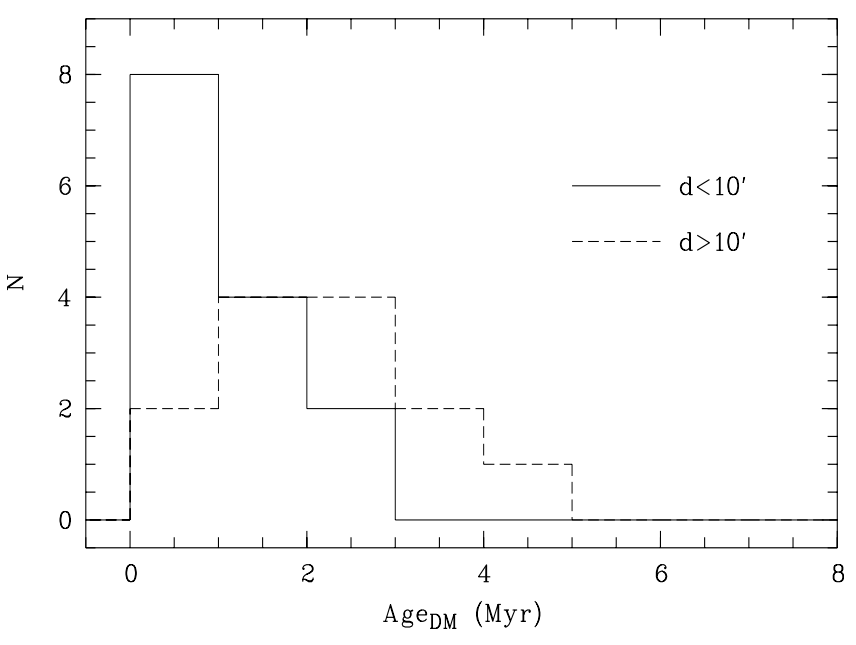

Fig. 11. Age distributions of the on-cloud (continuous line) and offcloud (dashed line) PMS stars in L1616. The ages are those derived from the D'Antona \& Mazzitelli (DM) tracks.

excess is more frequent among the on-cloud PMS stars than it is among the off-cloud PMS stars (see also Fig. 6 by Stanke et al. 2002), suggesting an age sequence.

In order to investigate whether there is any age difference between the visible on-cloud PMS stars and those distributed outside the cloud boundaries, we distinguish two groups, the dividing line being a 10 arcmin radius region (about $1.3 \mathrm{pc}$ at a distance of $450 \mathrm{pc}$ ) centered on the cometary cloud. The age distributions of the two groups of stars are shown in Fig. 11. 
There seems to be a trend for the on-cloud PMS stars to be slightly younger than those distributed outside the cloud. From a Kolmogorov-Smirnov test we find that the distributions are similar only at the $33 \%$ confidence level. However, the average ages are different to even less than the $1 \sigma$ level. Therefore, the trend seen in Fig. 11 is not statistically significant. We thus conclude that there is no age difference between the two groups of stars.

In any case, the millimeter source is definitely younger than the visible PMS stars. Considering a typical velocity dispersion of $1 \mathrm{~km} \mathrm{~s}^{-1}, 2$ Myr old stars would disperse over a distance of about $2 \mathrm{pc}$, which is the approximate projected size of the head of the cometary cloud. Therefore, it is likely that the on-cloud PMS stars that we see now are in reality surrounding the cloud, eastward of the millimeter source.

Hence, we conclude that there is evidence for two episodes of star formation in L1616: the 1-2 Myr old stars represent the first generation, while the millimeter source, and probably other not yet discovered embedded sources, partially surrounded by the 1-2 Myr old stars, represent the second generation.

With the present data, we cannot distinguish between the different triggering scenarios, but given the small age spread of the PMS stars and their spatial distribution relative to the head of the cometary cloud and embedded sources, as well as the cometary shape of the cloud and its high star formation efficiency, it is most likely that the star formation was induced by a single event. Either the impact of the winds of the massive stars of the Orion OB association or a single supernova explosion could have triggered the formation of the 1-2 Myr old stars and, later, the formation of embedded sources.

If the formation of the young stellar objects in L1616 was induced by a supernova explosion and there is an age difference of a few Myr between the millimeter source and the visible PMS stars, then the maximum separation of about $12 \mathrm{pc}$ would imply a progression velocity for the shock wave of the order of $10 \mathrm{~km} \mathrm{~s}^{-1}$, which is in line with the findings by Preibisch \& Zinnecker (1999) in the Upper Scorpius OB association. This rests, however, on the assumption that the PMS stars in L1616 are all at the same distance as the Orion SFR. Though the distance to L1616 may differ by no more than about $\pm 50 \mathrm{pc}$, this would be sufficient to have a substantial component of the triggering direction in the line of sight.

On the other hand, assuming that the millimeter source is about $0.4 \mathrm{Myr}$ old, the high-pressure shock front should be presently observed at a distance of about $4 \mathrm{pc}$ $\left(=0.4 \mathrm{Myr} \times 10 \mathrm{~km} \mathrm{~s}^{-1}\right)$ to the West of the cloud. An alternative scenario (Stanke et al. 2002) is that the supernova shock triggered only the formation of the oldest PMS stars. The youngest PMS were then formed by the impact of these stars.

The search for embedded sources to the West of the head of the cometary cloud will provide more clues to the star formation history in L1616.

\subsection{The Initial Mass Function in L1616}

It is interesting to check if the Initial Mass Function (IMF) of L1616 is consistent with that of the field. With the data we have, we cannot construct the IMF directly. However, by applying our completeness corrections provided in Table 6, we can make tentatively a comparison of the expected relative number of stars per mass bin with those provided by Miller \& Scalo (1979).

All the stars in our sample have masses less than $2.5 M_{\odot}$ and only 4 of them are more massive than $2 M_{\odot}$. These stars have magnitudes $R_{\mathrm{USNO}}<12 \mathrm{mag}$. Therefore, applying the completeness correction corresponding to this magnitude range, we expect a total of some $4 / 0.55 \approx 7$ stars with masses in the range $2<M / M_{\odot}<2.5$ in L1616. From the cumulative distribution by Miller \& Scalo (1979) (their Table 9), we compute that for the 7 stars with masses greater than $2 M_{\odot}$ we should expect about $21 \pm 4$ stars in the mass range $0.5<M / M_{\odot}<1$. Our sample contains 6 stars in this mass range, which have magnitudes $14<R_{\mathrm{USNO}}<15$. Applying the corresponding completeness correction we find that we should expect $6 / 0.26=23$ stars in that mass range, in agreement with the Miller \& Scalo (1979) IMF.

Doing the same exercise for another mass range we obtain the following. There are 13 stars with masses greater than $1 M_{\odot}$ in our sample, 4 of which more massive than $2 M_{\odot}$. These stars have magnitudes $R_{\mathrm{USNO}}<13$. Therefore, if we had observed the complete USNO sample with HYDRA, we would expect about $4 / 0.55+9 / 0.6 \approx 22$ stars with masses $1<M / M_{\odot}<2.5$ in L1616. Again, from the cumulative distribution by Miller \& Scalo (1979), we compute that, for the 22 stars with masses greater than $1 M_{\odot}$, we should expect about $28 \pm 5$ stars in the mass range $0.3<M / M_{\odot}<0.5$. Our sample contains 6 stars in this mass range, which have magnitudes $14<R_{\text {USNO }}<15$. Applying the corresponding completeness correction we find that there should be $6 / 0.26=23$ stars in that mass range.

Therefore, it seems that the IMF in L1616 is roughly consistent with the Miller \& Scalo (1979) IMF. The results are similar if we use the masses derived from the Palla \& Stahler (1999) tracks. However, due to the low number statistics, we consider these results to be preliminary. In order to establish definitely that the mass function in L1616 is consistent with that of the field, our unbiased lithium survey should be completed.

\subsection{Very low-mass PMS candidates in L1616}

The radiation and winds of OB stars or supernova shock waves that trigger star formation may also have an important impact on the mass accretion during the formation process. While in a $\mathrm{T}$ association a protostar may accumulate a significant fraction of mass, the mass accretion of a low-mass protostar in a region exposed to the winds of OB stars or supernova shock waves can be terminated abruptly because of the photoevaporation of the circumstellar accreting matter (Kroupa 2001, 2002). Therefore, many low-mass protostars never complete their accretion and hence may end up with sub-stellar masses. 


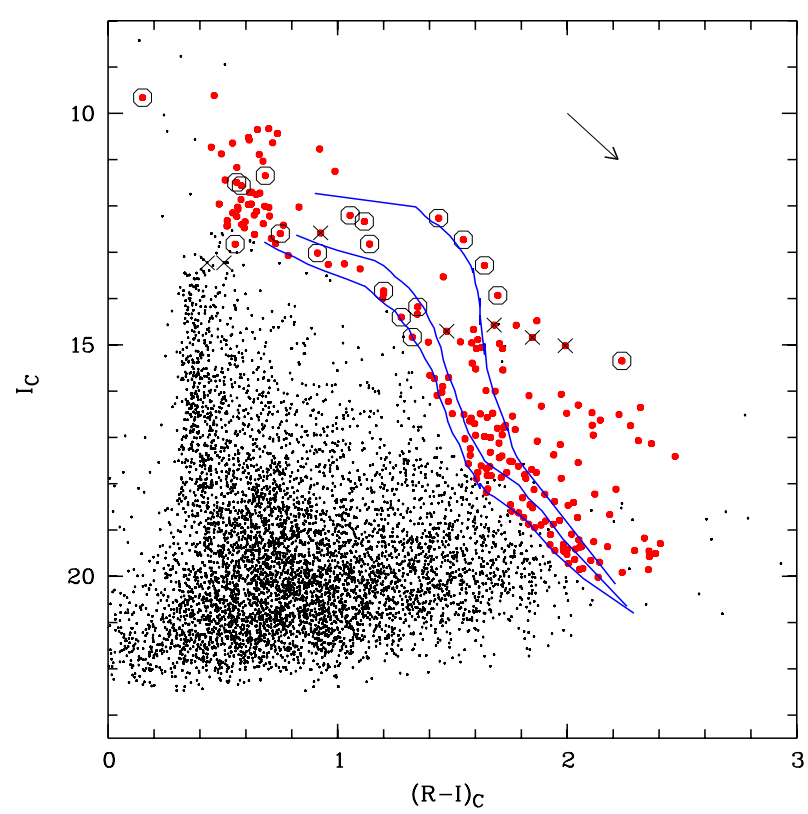

Fig. 12. $I_{\mathrm{C}}$ versus $\left(R_{\mathrm{C}}-I_{\mathrm{C}}\right)$ diagram for the point-like objects extracted from the $30^{\prime} \times 30^{\prime}$ WFI images in L1616.The continuous lines represent the theoretical PMS isochrones by Baraffe et al. (1998) for 1, 5 and $10 \mathrm{Myr}$, respectively, shifted to a distance modulus of 8.3 $(d=450 \mathrm{pc})$. The 18 low-mass PMS stars in the WFI images and HD 283915 are indicated with circles. The bigger dots represent the objects above the $10 \mathrm{Myr}$ isochrone. The $A_{V}=1 \mathrm{mag}$. reddening vector is also shown. The HRI X-ray sources detected with high confidence and which still lack optical spectroscopy are represented with " $\times$ " symbols.

These arguments have been proposed to explain the deficit of brown dwarfs (BDs) in The Taurus-Auriga association (Luhman 2000; Briceño et al. 2002) relative to the Orion nebula cluster (ONC) (Luhman et al. 2000). Recent studies of the young cluster IC 348, which is devoid of very massive stars, have also revealed a deficit of BDs relative to the ONC (Preibisch et al. 2003; Muench et al. 2003).

However, other processes different than the photoevaporation of circumstellar accreting matter may operate. For example, dynamical interactions in young multiple systems may lead to the ejection of objects before they can reach stellar masses (Reipurth \& Clarke 2001). The studies by Sterzik \& Durisen (2003) suggest ejection velocities for BDs of less than $2 \mathrm{~km} \mathrm{~s}^{-1}$. In about $1 \mathrm{Myr}$, such objects would move about $2 \mathrm{pc}$ (i.e. some $0.25^{\circ}$ at the distance of $450 \mathrm{pc}$ ) from their birth sites.

Our WFI data may provide more candidates for PMS objects, as well as very low-mass PMS stars and possible substellar objects in the core of the L1616 SFR. Based on colormagnitude diagrams and theoretical isochrones, we can select PMS candidates using the spectroscopically confirmed PMS stars to define the PMS locus in color-magnitude diagrams. In Fig. 12 the $I_{\mathrm{C}}$ versus $\left(R_{\mathrm{C}}-I_{\mathrm{C}}\right)$ diagram of the objects detected in the WFI images in the L1616 SFR is shown.

Excluding the new PMS stars, there are some 200 point-like objects which fall above the $10 \mathrm{Myr}$ isochrone. However, not all of these objects can be considered as PMS candidates, because many of them might be foreground or background stars. As discussed by Dolan \& Mathieu (2002), the resulting effect of the variable interstellar extinction of a cloud on background stars is to redden their colors, hence populating the same region of the color-magnitude diagrams as the PMS stars. Therefore, the only way to confirm the PMS nature of these objects is by means of future spectroscopic observations.

The faint end of the sequence shown in Fig. 12 may contain several young BDs. At the $2 \mathrm{Myr}$ age of the PMS stars in L1616, an object near the H-burning limit will have $(R-I) \sim$ 1.72 (Baraffe et al. 1998). By inspection of the color-magnitude diagram, we expect some 30-40 objects fainter than about $I=17$ and with $(R-I)>1.72$ which might be good candidates for BDs. Assuming that all the selected point-like objects above the $10 \mathrm{Myr}$ isochrone are PMS stars and that all of the 30-40 objects below $I=17$ are BDs, the fraction of sub-stellar objects would be about $18-25 \%$, which is rather high in comparison with the regions in Taurus and IC 348. However, due to the unknown nature of the selected objects, this estimate is very uncertain.

We can speculate on the number of BDs in L1616 using IMF models. From an extrapolation of the Miller \& Scalo (1979) IMF down to $0.08 M_{\odot}$ we estimate that, for the 22 stars with masses in the range $1<M / M_{\odot}<2.5$, some 12 very lowmass objects, near the sub-stellar boundary $\left(0.08<M / M_{\odot}<\right.$ 0.1 ), would be expected. However, below $0.1 M_{\odot}$ the Miller \& Scalo (1979) IMF may fail, because it is log-normal and hence, very likely underestimates the number of sub-stellar objects. If we use the Luhman et al. (2000) or Hillenbrand \& Carpenter (2000) IMF for the Trapezium cluster, a much larger number of sub-stellar objects (about 120) would be expected in L1616. However, our data alone do not allow to draw a firm conclusion yet. After being identified, we might expect the number of BDs in L1616 to be intermediate between Taurus (lower) and Trapezium (higher).

\section{Summary and conclusions}

We have performed a multi-wavelength study of the star forming region in the cometary cloud L1616. Our ROSAT-HRI $\mathrm{X}$-ray observations revealed $27 \mathrm{X}$-ray sources. For five of these sources we do not find any optical counterpart. The spectroscopic identification of the ROSAT-HRI observations and ROSAT BSC sources, combined with our unbiased lithium survey with HYDRA, allowed the identification of 22 new lowmass PMS stars, in addition to the eleven previously known young stellar objects in the region. Fifteen of the new PMS stars were X-ray detected, while the other seven were identified in our unbiased lithium survey with HYDRA. Based on the WFI images, we find that six additional ROSAT-HRI X-ray sources are very good candidates for PMS stars. Therefore, the number of young stellar objects known so far in L1616 is 33, but it might be as high as 40 .

From our multi-wavelength data, we derive the stellar parameters for 32 stars. Based on the level of X-ray emission,lithium content and kinematics, we confirm the PMS nature of these stars, as well as their association with L1616 as part of the Orion complex. By comparing the position of these stars in the HR diagram with PMS evolutionary tracks, we find that their age is about 1-2 Myr with a dispersion of about 
Table A.1. Additional PMS star candidates in L1616 and their photometry.

\begin{tabular}{|c|c|c|c|c|c|c|c|c|c|}
\hline $\mathrm{RXJ}$ & $\mathrm{RA}(2000)$ & $\operatorname{Dec}(2000)$ & $B$ & $V$ & $R_{\mathrm{C}}$ & $\overline{I_{\mathrm{C}}}$ & $J$ & $H$ & $K_{\mathrm{S}}$ \\
\hline $0506.8-0305$ & $\begin{array}{lll}05 & 06 & 48.98\end{array}$ & $\begin{array}{lll}-03 & 05 & 42.92\end{array}$ & 18.99 & 17.29 & 16.25 & 14.57 & 12.92 & 12.26 & 12.07 \\
\hline 0507.4-0317 & $\begin{array}{lll}05 & 07 & 25.93\end{array}$ & $\begin{array}{lll}-03 & 17 & 12.26\end{array}$ & 18.76 & 17.17 & 16.18 & 14.71 & 13.17 & 12.43 & 12.25 \\
\hline 0507.6-0318 & $\begin{array}{lll}05 & 07 & 37.67\end{array}$ & $\begin{array}{lll}-03 & 18 & 15.59\end{array}$ & 16.52 & 15.16 & 14.32 & 13.45 & 12.29 & 11.59 & 11.46 \\
\hline $0506.5-0320$ & $\begin{array}{lll}05 & 06 & 27.82\end{array}$ & $\begin{array}{lll}-03 & 20 & 27.02\end{array}$ & 19.86 & 18.12 & 17.01 & 15.02 & 13.18 & 12.60 & 12.30 \\
\hline $0506.4-0323$ & $\begin{array}{lll}05 & 06 & 25.98\end{array}$ & $\begin{array}{lll}-03 & 23 & 27.00\end{array}$ & 18.57 & 17.52 & 16.69 & 14.84 & 13.18 & 12.53 & 12.34 \\
\hline $0507.3-0326$ & $\begin{array}{lll}05 & 07 & 14.99\end{array}$ & $-03 \quad 26 \quad 47.31$ & 15.72 & 14.32 & 13.51 & 12.58 & 11.46 & 10.77 & 10.61 \\
\hline $0506.4-0328 \mathrm{~W}$ & $\begin{array}{lll}05 & 06 & 23.77\end{array}$ & $\begin{array}{lll}-03 & 28 & 47.88\end{array}$ & 15.18 & 14.26 & 13.73 & 13.23 & 13.07 & 12.64 & 12.55 \\
\hline $0506.4-0328 \mathrm{E}$ & $\begin{array}{lll}05 & 06 & 23.98\end{array}$ & $\begin{array}{lll}-03 & 28 & 45.94\end{array}$ & 14.69 & 14.04 & 13.66 & 13.23 & 12.80 & 12.52 & 12.43 \\
\hline
\end{tabular}

Table A.2. Parameters of PMS candidates in L1616.

\begin{tabular}{lccccrrrr}
\hline \hline RX J & SpT & $\log T_{\text {eff }}$ & $\log L / L_{\odot}$ & $\log L_{\mathrm{X}}$ & $\begin{array}{r}\text { Age (PS) } \\
{[\mathrm{Myr}]}\end{array}$ & $\begin{array}{r}\text { Age (DM) } \\
{[\mathrm{Myr}]}\end{array}$ & $\begin{array}{r}M(\mathrm{PS}) \\
{\left[M_{\odot}\right]}\end{array}$ & $\begin{array}{r}M(\mathrm{DM}) \\
{\left[M_{\odot}\right]}\end{array}$ \\
\hline $0506.8-0305$ & M4 & $3.517 \pm 0.015$ & $-0.72 \pm 0.11$ & $30.28 \pm 0.21$ & $1.05 \pm 0.90$ & $1.40 \pm 0.60$ & $0.20 \pm 0.07$ & $0.23 \pm 0.05$ \\
$0507.4-0317$ & M3 & $3.532 \pm 0.017$ & $-0.77 \pm 0.12$ & $30.02 \pm 0.25$ & $2.10 \pm 1.00$ & $1.95 \pm 0.95$ & $0.28 \pm 0.07$ & $0.29 \pm 0.07$ \\
$0507.6-0318$ & K7 & $3.618 \pm 0.018$ & $-0.43 \pm 0.02$ & $30.09 \pm 0.26$ & $6.50 \pm 1.50$ & $7.20 \pm 2.20$ & $0.85 \pm 0.10$ & $0.89 \pm 0.20$ \\
$0506.5-0320$ & M5 & $3.501 \pm 0.016$ & $-0.88 \pm 0.11$ & $31.05 \pm 0.06$ & $1.00 \pm 0.90$ & $1.50 \pm 0.70$ & $0.15 \pm 0.04$ & $0.17 \pm 0.05$ \\
$0506.4-0323$ & M4 & $3.517 \pm 0.018$ & $-0.85 \pm 0.01$ & $30.76 \pm 0.07$ & $1.80 \pm 0.80$ & $1.80 \pm 0.80$ & $0.20 \pm 0.08$ & $0.21 \pm 0.06$ \\
$0507.3-0326$ & K7 & $3.618 \pm 0.016$ & $-0.09 \pm 0.11$ & $30.46 \pm 0.14$ & $3.00 \pm 1.50$ & $1.80 \pm 0.90$ & $0.85 \pm 0.10$ & $0.78 \pm 0.15$ \\
$0506.4-0328 \mathrm{~W}$ & K0 & $3.712 \pm 0.011$ & $-0.33 \pm 0.03$ & $<29.99 \pm 0.20$ & - & - & - & - \\
$0506.4-0328 \mathrm{E}$ & G0 & $3.774 \pm 0.008$ & $-0.32 \pm 0.01$ & $<29.99 \pm 0.20$ & - & - & - & - \\
\hline
\end{tabular}

1 Myr. The small age dispersion can be explained in terms of efficient and quick star formation.

There is evidence for at least two episodes of star formation in L1616: the 1-2 Myr old stars represent the first generation, while the millimeter source and other possible embedded sources represent the second generation. Based on the spatial distribution of the PMS stars relative to the head of the cloud and the embedded source, as well as the cometary shape of the cloud and its high star formation efficiency, we believe that the star formation in L1616 was induced by a single event. Either the impact of the winds of the massive stars of the Orion OB association to the East of L1616 or a single supernova explosion could have triggered the formation of the 1-2 Myr old stars; later the jets and winds of the newly formed stars may have triggered the formation of the embedded sources.

The mass function in L1616 seems to be consistent with that of the field in the mass range $0.3<M / M_{\odot}<2.5$. However, since our unbiased lithium survey was not completed, the sample number statistics are poor and we consider this result as preliminary.

Acknowledgements. We thank the anonymous referee for his/her comments. We thank F. Palla, L. Testi and A. Natta for discussions. We also thank the Calar Alto staff, in particular F. Hoyo, M. Alises and A. Aguirre for their technical support. We also thank the WIYN telescope staff, in particular Gillian Rosenstein and Wendy Shook for their technical support during the observations. We also acknowledge A. Mejia for her assistantship during the HYDRA observations. We thank the ESO $2.2 \mathrm{~m}$ telescope team for their support during the WFI observations. This research has made use of the SIMBAD database, operated at CDS, Strasbourg, France. This research was conducted in part during the 2001 Research Experiences for Undergraduates (REU) Program at Cerro Tololo Inter-American Observatory (CTIO). The CTIO REU Program is funded by the National Science Foundation (NSF). CTIO is operated by AURA, Inc., under cooperative agreement with the NSF. This research has made use of the NASA/IPAC Infrared Science Archive, which is operated by the Jet Propulsion Laboratory, California Institute of Technology, under contract with the National Aeronautics and Space Administration.

\section{Appendix A: Additional candidates for PMS stars}

As pointed out at the end of Sect. 2.2.2, seven HRI X-Ray sources still lack spectroscopic observations. We used our WFI images in order to search for optical counterparts to these X-ray sources. For all of them we find a stellar counterpart, but for RX J0506.4-0328 two stars, with a separation of 3.7 arcsec, fall in the error circle. The 2MASS coordinates, as well as the optical and near-IR photometry for these stars are reported in Table A.1. The average photometric errors are the same as in Sect. 2.3.

As can be seen from the color-magnitude diagram in Fig. 12 these stars have magnitudes and colors consistent with those of the PMS stars in L1616.

Since all these stars are located relatively far from the core of the cluster, we do not expect a high interstellar extinction. Hence, we can attempt to assign spectral types to these stars, following the same procedure applied to Kiso A-097415, Kiso A-0974-17 and L1616 MIR4 as described in Sect. 3.5. The spectral types derived in this way are reported in Table A.2. 
Table B.1. Additional lithium stars found with HYDRA in the direction of L1616.

\begin{tabular}{|c|c|c|c|c|c|c|}
\hline 2MASS & $\mathrm{RA}(2000)$ & $\operatorname{Dec}(2000)$ & $\begin{array}{c}W(\mathrm{H} \alpha) \\
{[\AA]}\end{array}$ & $\begin{array}{r}W(\mathrm{Li}) \\
{[\mathrm{m} \AA]}\end{array}$ & $\mathrm{SpT}$ & $\begin{array}{r}R V \\
{\left[\mathrm{~km} \mathrm{~s}^{-1}\right]}\end{array}$ \\
\hline $05064458-0344422$ & $05 \quad 06 \quad 44.58$ & $-03 \quad 44 \quad 42.2$ & $-1.55 \pm 0.20$ & $70 \pm 10$ & M0 & $+12.0 \pm 4$ \\
\hline $05061320-0344394$ & $\begin{array}{lll}05 & 06 & 13.20\end{array}$ & $\begin{array}{lll}-03 & 44 & 39.4\end{array}$ & $4.10 \pm 0.50$ & $90 \pm 30$ & $\mathrm{G} 2$ & $+53.0 \pm 3$ \\
\hline $05075768-0342346$ & $\begin{array}{lll}05 & 07 & 57.68\end{array}$ & $-03 \quad 4234.6$ & $1.30 \pm 0.13$ & $90 \pm 30$ & $\mathrm{~K} 1$ & - \\
\hline $05054588-0327584$ & $\begin{array}{lll}05 & 05 & 45.88\end{array}$ & $\begin{array}{lll}-03 & 27 & 58.4\end{array}$ & $1.90 \pm 0.10$ & $130 \pm 20$ & K4 & $+16.0 \pm 3$ \\
\hline $05070351-0345027$ & $\begin{array}{lll}05 & 07 & 03.51\end{array}$ & $\begin{array}{lll}-03 & 45 & 02.7\end{array}$ & $2.70 \pm 0.20$ & $140 \pm 20$ & G6 & $+3.0 \pm 4$ \\
\hline $05055475-0332196$ & $\begin{array}{lll}05 & 05 & 54.75\end{array}$ & $\begin{array}{lll}-03 & 32 & 19.6\end{array}$ & $2.50 \pm 0.10$ & $110 \pm 15$ & F6 & $+0.0 \pm 3$ \\
\hline $05044592-0338227$ & $\begin{array}{lll}05 & 04 & 45.92\end{array}$ & $\begin{array}{lll}-03 & 38 & 22.7\end{array}$ & $1.40 \pm 0.20$ & $160 \pm 10$ & G8 & $+14.0 \pm 3$ \\
\hline $05060711-0305013$ & $\begin{array}{lll}05 & 06 & 07.11\end{array}$ & $\begin{array}{lll}-03 & 05 & 01.3\end{array}$ & $3.00 \pm 0.50$ & $140 \pm 20$ & $\mathrm{G} 2$ & $+44.0 \pm 3$ \\
\hline
\end{tabular}

Table B.2. Optical and NIR photometry for the additional lithium stars found with HYDRA.

\begin{tabular}{lccccccc}
\hline \hline 2MASS & $B$ & $V$ & $R_{\mathrm{C}}$ & $I_{\mathrm{C}}$ & $J$ & $H$ & $K_{\mathrm{S}}$ \\
\hline $05064458-0344422$ & - & - & - & - & 13.17 & 12.49 & 12.33 \\
$05061320-0344394$ & - & - & - & - & 10.69 & 10.44 & 10.39 \\
$05075768-0342346$ & - & - & - & - & 11.70 & 11.16 & 11.14 \\
$05054588-0327584$ & 14.48 & 13.45 & 12.92 & 12.22 & 11.68 & 11.16 & 11.00 \\
$05070351-0345027$ & - & - & - & - & 11.07 & 10.82 & 10.75 \\
$05055475-0332196$ & - & - & - & - & 11.73 & 11.54 & 11.53 \\
$05044592-0338227$ & - & - & - & - & 11.11 & 10.73 & 10.62 \\
$05060711-0305013$ & 13.17 & 12.89 & 12.79 & 12.23 & 11.82 & 11.50 & 11.48 \\
\hline
\end{tabular}

Also, none of the stars show evidence for strong infrared excess. Therefore, we can also attempt to determine their luminosity by adopting a distance of $450 \mathrm{pc}$. The spectral type versus effective temperature relation for luminosity class $V$ by de Jager \& Nieuwenhuijzen (1987) were used to estimate effective temperatures based on the putative spectral types. The bolometric luminosities are derived in the same way as described in Sect. 3.6 and the X-ray luminosities are computed as described in sect. 3.7. The results are listed in Table A.2.

The comparison of the position of these stars in the HR diagram with the PMS tracks by D'Antona \& Mazzitelli (1997) and by Palla \& Stahler (1999) shows that, except for RX J0506.4-0328W and RX J0506.4-0328E, the other six stars have masses and ages, as well as X-ray luminosities which are quite consistent with those of the PMS stars in L1616. The results are reported in Table A.2.

We therefore conclude that the first six stars listed in Table A.1 are very likely low-mass PMS stars, members of L1616. In this case, the number of young stellar objects in L1616 would increase to 39.

\section{Appendix B: Additional lithium stars found with HYDRA}

Based on our HYDRA observations, we have found 8 lithium stars in addition to the 22 new PMS stars discussed in the text. All these stars have lithium equivalent widths $W(\mathrm{Li})<$ $200 \mathrm{~m} \AA$. Therefore, they are most probably ZAMS stars. These objects are listed in Table B.1 together with their $\mathrm{H} \alpha$ and $\mathrm{Li}$ equivalent widths, spectral types and radial velocity estimates, performed in the same way as described in Sect. 3 .

The optical and 2MASS NIR magnitudes for these stars are reported in Table B.2. Only two of these stars fall within the area of our WFI images. The average errors in the photometry are the same as in Sect. 2.3.

\section{References}

Alcalá, J. M., Krautter, J., Schmitt, J. H. M. M., et al. 1995, A\&AS, 114,109

Alcalá, J. M., Krautter, J., Covino, E., et al. 1997, A\&A, 319, 184

Alcalá, J. M., Covino, E., Torres, G., et al. 2000a, A\&A, 353, 186

Alcalá, J. M., Covino, E., Sterzik, M. F., et al. 2000b, A\&A, 355, 629

Alcalá, J. M., Radovich, M., Silvotti, R., et al. 2002, SPIE, 4836, 406

Baraffe, I., Chabrier, G., Allard, F., \& Hauschildt, P. H. 1998, A\&A, 337,403

Bessell, M. S., \& Wood, P. R. 1984, PASP, 96, 247

Bessell, M. S., \& Brett, J. M. 1988, PASP, 100, 1134

Boss, A. P. 2003, in Galactic Star Formation Across the Stellar Mass Spectrum, Proc. International Astronomical Observatories in Chile workshop, held 11-15 March 2002 at La Serena, Chile. ed. J. M. De Buizer, \& N. S. van der Bliek (San Francisco: Astronomical Society of the Pacific), ASP Conf. Ser., 287, 281

Briceño, C., Luhman, K. L., Hartmann, L., Stauffer, J. R., \& Kirkpatrick, J. D. 2003, IAUS, 211, 81

Carpenter, J. M. 2001, AJ, 121, 2851

Cieslinski, D., Jablonski, F. J., \& Steiner, J. E. 1997, A\&AS, 124, 55

Covino, E., Alcalá, J. M., Allain, S., et al. 1997, A\&A, 328, 187 
Cruddace, R. G., Hasinger, G. R., \& Schmitt, J. H. 1988, in Astronomy from large databases: Scientific objectives and methodological approaches; Proc. Conf., Garching, Federal Republic of Germany, Oct. 12-14, 1987 (A89-27176 10-82). Garching, Germany, European Southern Observatory, 177

D’Antona, F., \& Mazzitelli, I. 1997, Mem. Soc. Astron. It., 68, 807

de Jager, C., \& Nieuwenhuijzen, H. 1987, A\&A, 177, 217

Dolan, C. J., \& Mathieu, R. D. 1999, AJ, 121, 2124

Dolan, C. J., \& Mathieu, R. D. 2002, AJ, 123, 387

Elmegreen, B. G., Kimura, T., Tosa, M. 1995, ApJ, 451, 675

Feigelson, E. D., Casanova, S., Montmerle, Th., \& Guibert, J. 1993, ApJ, 416, 623

Feigelson, E. D. 1996, ApJ, 468, 306

Flaccomio, E., Damiani, F., Micela, G., et al. 2003, ApJ, 582, 398

Frasca, A., Alcalá, J. M., Covino, E., et al. 2003, A\&A, 405, 149

Gagne, M., Caillault, J.-P., \& Stauffer, J. R. 1995, ApJ, 445, 280

Hartigan, P., Strom, K. M., \& Strom, S. E. 1994, ApJ, 427, 961

Hillenbrand, L. A., \& Carpenter, J. M. 2000, ApJ, 540, 236

Huensch, M., Schmitt, J. H. M. M., Sterzik, M. F., \& Vogues, W. 1999, A\&AS, 135, 319

Kroupa, P. 2001, MNRAS, 322, 231

Kroupa, P. 2002, Science, 295 (5552), 82

Lada, C. J., Elmegreen, B. G., \& Blitz, L. 1978, in Protostars and planets: Studies of star formation and of the origin of the solar system (Tucson, Ariz: University of Arizona Press), 341

Lada, C. J. 1987 in Star formation: from OB Associations to protostars, ed. M. Peimbert \& J. Jugaku (Dordrecht: D. Reidel), Proc. IAU Symp., 115, 1

Luhman, K. L. 2000, ApJ, 544, 1044

Luhman, K. L., Rieke, G. H., Young, E. T., et al. 2000, ApJ, 540, 1016 Lynds, B. T. 1962, ApJS, 7, 1

Maddalena, R. J., Morris, M., Moscowitz, J., \& Thaddeus, P. 1986, ApJ, 303, 375

Mamajek, E. E., Meyer, M. R., \& Liebert, J. 2002, AJ, 124, 1670

Martín, E. L., \& Magazzù, A. 1999, A\&A, 342, 173

Miller, G. E., \& Scalo, J. M. 1979, ApJS, 41, 513

Monet, D., Bird, A., \& Canzian, B., et al. 1998, US Naval Observatory Flagstaff Station (USNOFS) and Universities Space Research Association (USRA) stationed at USNOFS
Muench, A. A., Lada, E. A., Lada, C. J., et al. 2003, AJ, 125, 2029

Nakano, M., Kogure, T., \& Wiramihardja, S. 1995, PASJ, 48, 889

Neckel, T., \& Chini, R. 1980, A\&AS, 39, 411

Ogura, K., \& Sugitani, K. 1998, PASA, 15, 91

Paresce, F. 1984, AJ, 89, 1022

Pavlenko, Y. V., \& Magazzù, A. 1996, A\&A, 311, 961

Palla, F., \& Stahler, S. W. 1999, ApJ, 525, 772

Pfeiffer, M. J., Frank, C., Baumüller, D., Fuhrmann, K., \& Gehren, T. 1998, A\&AS, 130, 381

Preibisch T., Günther E., Zinnecker H., et al. 1998, A\&A, 333, 619

Preibisch, Th., \& Zinnecker, H. 1999, AJ, 117, 2381

Preibisch, T., Stanke, T., \& Zinnecker, H. 2003, A\&A, 409, 147

Ramesh, B. 1995, MNRAS, 276, 923

Randich, S., Aharpour, N., Pallavicini, R., Prosser, C. F., \& Stauffer, J.R. 1997, A\&A, 323, 86

Reipurth, B., \& Clarck, C. 2001, AJ, 122, 432

Stanke, T., Smith, M. D., Gredel, R., \& Szokoly, G. 2002, A\&A, 393, 251

Sterzik, M. F., Alcalá, J. M., Neuhäuser, R., \& Schmitt, J. H. M. M. 1995, A\&A, 297, 418

Sterzik, M. F., Alcalá, J. M., Neuhäuser, R., \& Durisen, R. H. 1998, in The Orion complex revisited, ed. A. Burkert, \& M. McCaughrean, PASP Conf. Ser., in press

Sterzik, M. F., \& Durisen, R. H. 2003, A\&A, 400, 1031

Stetson, P. B. 1987, PASP, 99, 191

Tripicchio, A., Severino, G., Covino, E., Terranegra, L., \& García López, R. J. 1997, A\&A, 327, 681

Tripicchio, A., Alcalá, J.M., Covino, E., \& D’Antona, F. 2000, Mem. S. A. It., 71, 1021

Vrba, F. J., \& Rydgren, A. E. 1985, AJ, 90, 1490

Walter, F. M., Vrba, F. J., Mathieu, R. D., Brown, A., \& Myers, P. C. 1994, AJ, 107, 692

Walter, F. M. 1996, ASP Conf. Ser., 109, Proc. 9th Cambridge workshop held 3-6 October 1995 in Florence, Italy, ed. R. Pallavicini \& A. K. Dupree, 441

Walter, F. M., Alcalá, J. M., Neuhauser, R., Sterzik, M., \& Wolk, S. J. 2000, Protostars and Planets IV, ed. V. Mannings, A. P. Boss, \& S. S. Russell (University of Arizona Press), 273 